\title{
Experimental study of the turbulence intensity effects on marine current turbines behaviour. Part I: One single turbine
}

\author{
Paul Mycek $^{\mathrm{a}, \mathrm{b}}$, Benoît Gaurier ${ }^{\mathrm{b}}$, Grégory Germain $^{\mathrm{b}}$, Grégory Pinon $^{\mathrm{a}, *}$, Elie Rivoalen ${ }^{\mathrm{a}, \mathrm{c}}$ \\ a Laboratoire Ondes et Milieux Complexes, UMR 6294, CNRS e Université du Havre, 53, rue de Prony, BP 540, \\ F-76058 Le Havre Cedex, France \\ ${ }^{b}$ IFREMER, Marine Structures Laboratory, 150, quai Gambetta, BP 699, F-62321 Boulogne-Sur-Mer, France \\ ${ }^{\mathrm{c}}$ Laboratoire d'Optimisation et Fiabilité en Mécanique des Structures, EA 3828, INSA de Rouen, Avenue de \\ I'Université, BP 08, F-76801 Saint-Etienne-du-Rouvray, France \\ *: Corresponding author : Grégory Pinon, email address : gregory.pinon@univ-lehavre.fr \\ paul.mycek@ifremer.fr ; benoit.gaurier@ifremer.fr ; gregory.germain@ifremer.fr ; elie.rivoalen@insa-rouen.fr
}

\begin{abstract}
:
The ambient turbulence intensity in the upstream flow plays a decisive role in the behaviour of horizontal axis marine current turbines.

Experimental trials, run in the IFREMER flume tank in Boulogne-Sur-Mer (France) for two different turbulence intensity rates, namely $3 \%$ and $15 \%$, are presented. They show, for the studied turbine configuration, that while the wake of the turbine is deeply influenced by the ambient turbulence conditions, its mean performances turn out to be slightly modified.
\end{abstract}

The presented conclusions are crucial in the view of implanting second generation turbines arrays. In addition, complete and detailed data sets (wake profiles and performance graphs) are made available to the scientific community in order to encourage further comparisons.

\section{Highlights}

Trials on 3-bladed horizontal axis marine current turbine were run in a flume tank. $>$ Two ambient turbulence intensity rates are considered. The wake and performances of the turbine are characterised.

- The ambient turbulence intensity deeply influences the behaviour of the turbine.

Keywords : Marine current turbine ; Performance ; Wake ; Turbulence ; Array ;Tidal turbine

\section{Introduction}

The ambient turbulence intensity in the upstream flow plays a decisive role in the behaviour of horizontal axis marine current turbines. First, turbulence intensity may influence the turbine performances but, probably most important, it deeply influences the wake shape. This last issue is of crucial matter for the onset of marine current turbine arrays. Indeed, in second generation arrays, the wake of an upstream turbine may irreparably affect the power performances of another turbine positioned downstream. This aspect of elementary interactions between marine current turbines in an array will be treated in the second part of this study [1]. The present paper aims at characterising precisely the performances and wake of a single turbine depending on the ambient 
turbulence intensity of the incoming flow. The results of this first part will represent a strong basis for comparisons with the twin turbines setups investigated in the second part.

Several in situ studies were carried out to characterise the turbulence intensity in potential sites, where marine current turbines are expected to be installed. These studies are extremely difficult to undertake owing to their important cost, the possible harsh metocean conditions encountered, as well as the high quality measurement devices that are required to assess the turbulence intensity. Most of the studies focused on a streamwise turbulence intensity $I_{\infty}^{1 D}=\sigma_{u} / U_{\infty}$. In order to obtain a 3D turbulence intensity rate $I_{\infty}$, Milne et al. [2] precisely measured the anisotropic ratio $\left(\sigma_{u}: \sigma_{v}: \sigma_{w}\right)=(1: 0.75: 0.56)$ in the Sound of Islay (Scotland, UK); and found it similar to the values given by Nezu et Nakagawa [3]. Table 1 summarises the in situ flow measurements mentioned in the sequel. In order to obtain 3D $I_{\infty}$ values from the $I_{\infty}^{1 D}$ given in all other studies, the precise $\left(\sigma_{u}: \sigma_{v}: \sigma_{w}\right)=(1: 0.75: 0.56)$ anisotropic ratio from [2] was assumed.

\begin{tabular}{lcccccc}
\hline Location & $I_{\infty}^{1 D}[\%]$ & $I_{\infty}[\%]$ & $U_{\infty}[\mathrm{m} / \mathrm{s}]$ & $z_{m}[\mathrm{~m}]$ & Techniques & Ref \\
\hline Fall of Warness & $10-11$ & $7.9-8.7$ & 1.5 & 5.0 & ADCP & {$[4,5]$} \\
Sound of Islay & $12-13$ & $9.5-10.3$ & 2.0 & 5.0 & ADV & {$[2]$} \\
Puget Sound & $8.4 / 11.4$ & $6.6 / 9.0$ & $1.3( \pm 0.5)$ & 4.7 & ADV / ADCP & {$[6]$} \\
Strangford Narrows & $4-9$ & $3.2-7.1$ & $1.5-3.5$ & 14 & - & {$[7]$} \\
East River, NY & $20-30$ & $16-24$ & $1.5-2.3$ & 5.22 & ADCP & {$[8]$} \\
\hline
\end{tabular}

Table 1: In situ measurements of turbulence intensity rates $I_{\infty}$ in the literature. $I_{\infty}^{1 D}$ stands for the streamwise turbulence intensity rate, $I_{\infty}$ the $3 \mathrm{D}$ turbulence intensity rate, $U_{\infty}$ the mean velocity, $z_{m}$ the vertical distance to the seabed. Except for the study of Milne et al. [2], where the anisotropic ratio $\left(\sigma_{u}: \sigma_{v}: \sigma_{w}\right)=(1: 0.75: 0.56)$ was measured, this precise ratio was assumed for all the other studies, so as to deduce $3 \mathrm{D} I_{\infty}$ values.

Among the last studies, Osalusi et al. $[4,5]$ used an Acoustic Doppler Current Profiler (ADCP) to assess several turbulence characteristics such as Turbulent Kinetic Energy production and dissipation, Reynolds shear stresses, etc. Their study was carried out in the Fall of Warness (Orkney Islands, Scotland, UK) during a week, precisely at the tidal test site of the European Marine Energy Centre (EMEC). Following the previous assumption, the 3D turbulence intensity lies between $I_{\infty} \approx 7.9-8.7 \%$ at 5 meters from the seabed for a mean velocity of $1.5 \mathrm{~m} \cdot \mathrm{s}^{-1}$. In their in situ study, Milne et al. found a 3D turbulence intensity $I_{\infty}$ of approximately $9.5-10.3 \%$, depending on flood and ebb tides. The measurements [2] were performed in the Sound of Islay (Scotland, UK) at 5 meters from the seabed for a mean velocity of $2.0 \mathrm{~m} \cdot \mathrm{s}^{-1}$. They lasted approximately 15 days and used a $4 \mathrm{~Hz}$ ADV (Acoustic Doppler Velocimeter). Thomson et al. [6] carried out a similar study comparing two measurement techniques, $\mathrm{ADCP}$ and $\mathrm{ADV}$, at $4.7 \mathrm{~m}$ from the seabed in the Puget Sound (Washington State, US). They clearly indentified several sources of error while measuring turbulence intensities with ADCP, and precisely quantified them. ADCP and AVD techniques respectively gave a streamwise turbulent intensity of $I_{\infty}^{1 D}=8.4 \%$ and $I_{\infty}^{1 D}=11.4 \%$ at the same point (the Nodule Point, Puget Sound), even with Doppler noise correction for the ADCP measurements. It should be mentioned that although ADCPs are commonly used to evaluate turbulence, potential errors can be made, essentially due to the compromise made between accuracy and resolution. Errors may also issue from the hypothesis made on the steadiness and horizontal uniformity of the turbulence, which are in contradiction with the highly intermittent and multi-scale feature of the phenomenon $[9,10]$.

The streamwise turbulence intensities from the three previous studies $[2,4-6]$ are very similar in order of magnitude, ranging from approximately $8.4 \%$ to $13 \%$. However, the turbulence intensity $I_{\infty}$ does not seem to be a global constant, or even a geographical site constant. As a matter of fact, 
in a recent paper, Mac Enri et al. [7] indicate that the 3D turbulence intensity $I_{\infty}$ may vary from approximately $3.2 \%$ to $7.1 \%$ depending on the mean velocity, ebb and flood or neap and spring tides. At a given point, the turbulence intensity may vary significantly depending on time varying tidal physical values. Their measurements were carried out in the Strangford Narrows (Republic of Ireland), where the 1.2 MW SeaGen marine current turbine is installed. The measurements were performed at the hub level, i.e. 14 meters from the seabed, with an electromagnetic current meter (Valeport Model 803) with a $1 \mathrm{~Hz}$ frequency. Their velocity measurements were also calibrated with an ADCP.

Spatial variations may also occur in a given geographical site. The study by Gooch et al. [11] gave varying turbulent intensity rates depending on the precise location in the Puget Sound. In fact, five locations in the Puget Sound were assessed: Admiralty Inlet, North East off the Marrowstone Lighthouse and three locations East the Marrowstone Island at the smallest cross-sectional area of the Puget Sound. The measurements, from one to two months (between 33 and 75 days), were obtained with ADCP. Small variations between ebb and flood tides were observed. However, the streamwise turbulent intensities obtained were ranging from $I_{\infty}^{1 D} \approx 2.8$ to about $5.4 \%$ at several locations and several depths within the Puget Sound. Taking the latest results by Thomson et al. [6] into account, for a single geographical site, the streamwise turbulence intensity ratio $I_{\infty}^{1 D}$ range lies between about $2.8 \%$ and $11.8 \%$ at different locations and different depths. Even if the turbulence intensity increases with the depth [6], these variations are however important within a single geographical site. Finally, the study by Li et al. [8] in the East River (New York, NY) gave an estimated $I_{\infty} \approx 16-24 \%$ provided that the $\left(\sigma_{u}: \sigma_{v}: \sigma_{w}\right)$ assumed ratio is still valid in the case of a river. Still, their measurements of streamwise turbulence intensity rate $I_{\infty}^{1 D}=20-30 \%$ are noticeably higher than the previous ones from Table 1.

The flume tank experiments presented in this paper were carried out in incoming flows with two precise turbulence intensity rates, namely $I_{\infty}=3 \%$ and $I_{\infty}=15 \%$. These two values are actually representative of the $I_{\infty}$ range depicted in Table 1, with the minimum values of $I_{\infty} \approx 3.2 \%$ in the Strangford Narrows [7] to the higher values of Li et al. [8] in the East River $\left(I_{\infty} \gtrsim 15 \%\right)$. For the given turbine geometry used in this study, the present paper aims at describing major differences in the performance and wake characteristics between $I_{\infty}=3 \%$ and $15 \%$.

Experimental trials on a single marine current turbine in a flume tank have already been carried out, using different techniques. Bahaj et al. $[12,13]$ carried out a power $\left(C_{P}\right)$ and thrust $\left(C_{T}\right)$ coefficient study on a $0.8 \mathrm{~m}$-diameter turbine model in a towing tank and in a cavitation tunnel. However, in the previous two studies, the wake behind the turbine was not characterised. On the other hand, experimental wake characterisation is available in $[14,15]$ under an actuator disc approximation. Unfortunately, this approximation does not take intrinsically into account the fluid rotation in the wake, and the power and thrust assessment is more complex. Rose et al. [16] performed several experimental trials, some in a flume tank and others in open water (Montgomery Lough) using either PIV (Particle Image Velocimetry) or ADV (Acoustic Doppler Velocimeter) techniques. Several turbines were tested, the biggest one being a $1 / 10^{\text {th }}$ scale turbine model of $1.5 \mathrm{~m}$ in diameter in the lake (Montgomery Lough). However, only wake velocity measurements are presented in the paper without any turbine performance. Stallard et al. [17] also give interesting information on a single turbine wake, including turbulence intensity, even if their study mainly deals with turbine interactions. For Tedds et al. [18], many turbine performance curves are depicted depending on the number of blades, pitch angles, etc. but without much details about the wake velocity profiles. In another study, Milne et al. [19] gave interesting turbine performance and thrust 
curves even though their study was mainly oriented towards blade loads owing to oscillatory flows, similarly to Davies et al. [20].

The present study aims at characterising both the power and thrust coefficient curves $\left(C_{P}\right.$ and $C_{T}$ curves) together with detailed wake profiles including turbulence intensities. This paper follows the same experimental procedures as presented in [21], but with an open-modified version of the turbine which enables the diffusion of the blades geometry. Some of the experimental results were partially presented or used as a matter of numerical-experimental validation in [22, 23]. The present document presents all our latest experimental results of a single 3-bladed turbine immersed in two different turbulence intensities, namely $I_{\infty}=3 \%$ and $I_{\infty}=15 \%$.

First of all, section 2 details the experimental setup, measurement techniques and turbine geometry. Section 3 presents the power and thrust coefficient curves, for different incoming mean velocities and the two turbulence intensity rates. The standard deviations of these curves are also shown. Then, section 4 gives streamwise velocity, turbulence intensity and Reynolds shear stress maps depending on the two turbulence intensity rates. The wake is also characterise using integrated quantities. Finally, most of the raw results are made available in the appendices as a matter of validation with future numerical studies. This intends to answer to a recurrent request, the latest being by Churchfield et al. [24].

\section{Experiments description}

This section aims at giving a detailed description of the experimental setup and measurement facilities used for the experiments. The definitions of the quantities that will be used for the analysis are also presented in this section.

\subsection{Flume tank and experiment description}

The trials were run in the IFREMER (French Research Institute for Exploitation of the Sea) wave and current flume tank, depicted in Figure 1. The flume tank working section is $18 \mathrm{~m}$ long by $4 \mathrm{~m}$ large and $2 \mathrm{~m}$ deep. The streamwise flow velocity range is 0.1 to $2.2 \mathrm{~m} \cdot \mathrm{s}^{-1}$. More details about the flume tank can be found in [25].

The experimental setup is illustrated in Figure 2, where Cartesian coordinate system is considered, with the rotor centre as its origin $O(0,0,0)$ and $\mathbf{e}_{x}, \mathbf{e}_{y}$ and $\mathbf{e}_{z}$ as unit vectors. The flow velocity field is denoted by $\mathbf{u}$ and is function of the position $\mathbf{x}$ and time $t$ at which it is measured. The position and velocity components are respectively denoted by $x, y, z$, and $u, v, w$ :

$$
\begin{aligned}
& \mathbf{x}=x \mathbf{e}_{x}+y \mathbf{e}_{y}+z \mathbf{e}_{z}=(x, y, z) \\
& \mathbf{u}=u \mathbf{e}_{x}+v \mathbf{e}_{y}+w \mathbf{e}_{z}=(u, v, w)
\end{aligned}
$$

For convenience, those components may as well be referred to in their indicial notation as $x_{i}$ and $u_{i}$ respectively, with $i$ from 1 to 3 .

\subsection{Reynolds decomposition}

The flow velocity field is broken down thanks to Reynolds decomposition:

$$
\mathbf{u}(\mathbf{x}, t)=\overline{\mathbf{u}}(\mathbf{x})+\mathbf{u}^{\prime}(\mathbf{x}, t)
$$




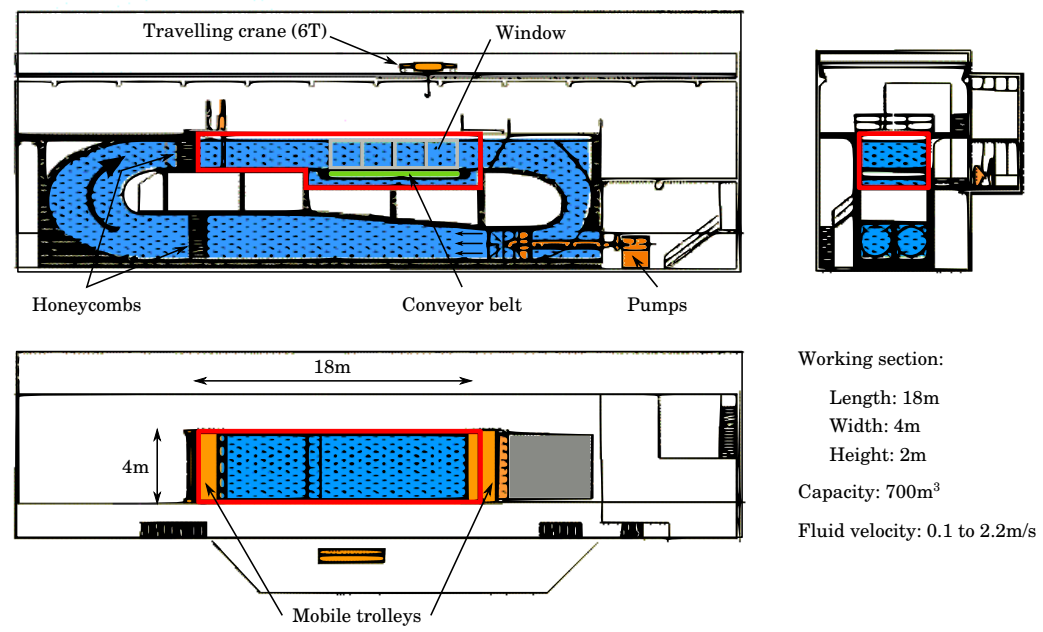

Figure 1: IFREMER's Boulogne-sur-Mer flume tank description.

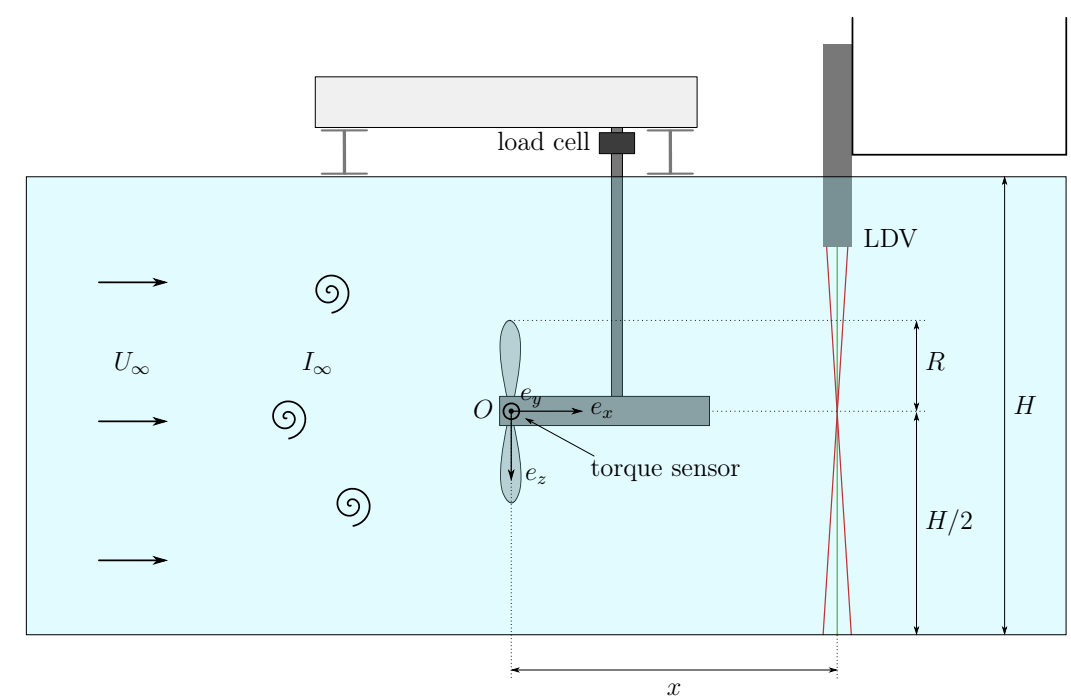

Figure 2: Schematic side view of the experimental setup. The origin $O(0,0,0)$ is chosen at the rotor centre.

where $\overline{\mathbf{u}}$ is the time average of $\mathbf{u}$, defined by:

$$
\overline{\mathbf{u}}(\mathbf{x})=\frac{1}{T} \int_{0}^{T} \mathbf{u}(\mathbf{x}, t) d t
$$

where $[0 ; T]$ is the averaging period. Thus, $\overline{\mathbf{u}}$ represents the steady part of the velocity, while $\mathbf{u}^{\prime}$ represents its fluctuating part. The components $R_{i j}$ of the Reynolds stress tensor $R$ are defined as follows:

$$
R_{i j}=\overline{u_{i}^{\prime} u_{j}^{\prime}}=\overline{\left(u_{i}-\bar{u}_{i}\right)\left(u_{j}-\bar{u}_{j}\right)} \quad i, j=1, \ldots, 3 .
$$


The diagonal elements $R_{i i}=\overline{u_{i}^{\prime 2}}$ are denoted by $\sigma^{2}(u), \sigma^{2}(v)$ and $\sigma^{2}(w)$ in analogy with the variance in statistics.

\subsection{Upstream flow conditions}

The upstream turbulence intensity rate $I_{\infty}$ is defined by:

$$
I_{\infty}=100 \sqrt{\frac{\frac{1}{3}\left[\sigma^{2}\left(u_{\infty}\right)+\sigma^{2}\left(v_{\infty}\right)+\sigma^{2}\left(w_{\infty}\right)\right]}{\bar{u}_{\infty}^{2}+\bar{v}_{\infty}^{2}+\bar{w}_{\infty}^{2}}}
$$

where the velocity components $u_{\infty}, v_{\infty}, w_{\infty}$ are those of the upstream velocity $\mathbf{u}_{\infty}$. The LDV measurements presented in the sequel are performed in the $x O y$ plane, the $2 \mathrm{D}$ upstream turbulence intensity rate is thus defined as follows:

$$
I_{\infty}^{2 D}=100 \sqrt{\frac{\frac{1}{2}\left[\sigma^{2}\left(u_{\infty}\right)+\sigma^{2}\left(v_{\infty}\right)\right]}{\bar{u}_{\infty}^{2}+\bar{v}_{\infty}^{2}}} .
$$

Similarly, this $2 \mathrm{D}$ quantity can be computed in the $x O z$ plane as well, but it is irrelevant for the results presented hereafter.

In the present study, two different ambient turbulence conditions are considered. The turbulence in the flume tank is induced by the current generation process. As a matter of fact, without the use of a honeycomb, the natural ambient turbulence intensity of the flow is about $I_{\infty}=15 \%$. A smoother flow may be obtained by the use of honeycombs and the turbulence intensity rate then reduces to about $I_{\infty}=3 \%$. In the sequel, those two turbulence conditions will simply be referred to as $I_{\infty}=15 \%$ (without honeycombs) and $I_{\infty}=3 \%$ (with honeycombs). These values were obtained by LDV measurements of $(u, v)$ and $(u, w)$ at different points in the area swept by the turbine blades.

\begin{tabular}{ccccccc}
\hline$U_{\infty}\left[\mathrm{m} \cdot \mathrm{s}^{-1}\right]$ & $I_{\infty}[\%]$ & $I_{\infty}^{2 D}[\%]$ & & $U_{\infty}\left[\mathrm{m} \cdot \mathrm{s}^{-1}\right]$ & $I_{\infty}[\%]$ & $I_{\infty}^{2 D}[\%]$ \\
\cline { 1 - 3 } & 2.7 & 3.2 & & 0.41 & 15.3 & 14.6 \\
0.6 & 2.6 & 2.7 & & 0.62 & 14.7 & 13.0 \\
0.8 & 2.7 & 3.1 & & 0.83 & 14.7 & 13.3 \\
1.0 & 2.7 & 3.1 & & 1.03 & 14.7 & 14.0 \\
\hline
\end{tabular}
(a) $I_{\infty}=3 \%$.
(b) $I_{\infty}=15 \%$.

Table 2: Measured values of $I_{\infty}$ and $I_{\infty}^{2 D}$ for the indicative turbulence conditions $I_{\infty}=3 \%$ (left) and $I_{\infty}=15 \%$ (right), together with different upstream velocity conditions. These values were obtained by LDV measurements at one point, placed approximately at the location of the turbine.

Table 2 presents measured values of $I_{\infty}$ and $I_{\infty}^{2 D}$ for different upstream flow conditions: the above mentioned turbulence intensity conditions $I_{\infty}=3 \%$ (Table 2a) and $I_{\infty}=15 \%$ (Table 2b), together with different upstream velocity conditions $U_{\infty}$. Whatever the upstream velocity $U_{\infty}$, for a given $I_{\infty}$ condition, the measured values of $I_{\infty}$ or $I_{\infty}^{2 D}$ remain close to each other. Moreover, the measured $I_{\infty}$ is always close to the indicative $I_{\infty}$ value $(3 \%$ or $15 \%)$. However, it should be stressed that the corresponding $2 D$ turbulence intensity $I_{\infty}^{2 D}$ slightly differs from the indicative values of $3 \%$ or $15 \%$. In particular, with $I_{\infty}=15 \%$, the $I_{\infty}^{2 D}$ value are significantly below their $3 \mathrm{D}$ counterparts, more precisely $I_{\infty}^{2 D} \approx 13 \%$. This is due to the omission of the $z$-components $\bar{w}_{\infty}$ 
and $\sigma\left(w_{\infty}\right)$ in the $2 D$ evaluation, whose influence is non-negligible in the $I_{\infty}=15 \%$ turbulence condition. This remark justifies the use of $I_{\infty}^{2 D}$ as a comparison basis when studying the wake in terms of downstream turbulence intensity (cf. section 4), which is measured in $2 D$ (cf. section 2.7).

The mean upstream axial velocity $\bar{u}_{\infty}$ may be simply denoted by $U_{\infty}$. Five different upstream velocities (from $U_{\infty}=0.4$ to $1.2 \mathrm{~m} \cdot \mathrm{s}^{-1}$ ) are considered with $I_{\infty}=3 \%$, while four velocities (from $U_{\infty}=0.41$ to $1.03 \mathrm{~m} \cdot \mathrm{s}^{-1}$ ) are considered with $I_{\infty}=15 \%$. Figure 3 gives the profiles of the $u_{\infty}$ and $v_{\infty}$ upstream velocity components across the tank, in terms of mean value and standard deviation, for both considered turbulence intensity rates and $U_{\infty} \approx 0.8 \mathrm{~m} \cdot \mathrm{s}^{-1}$.
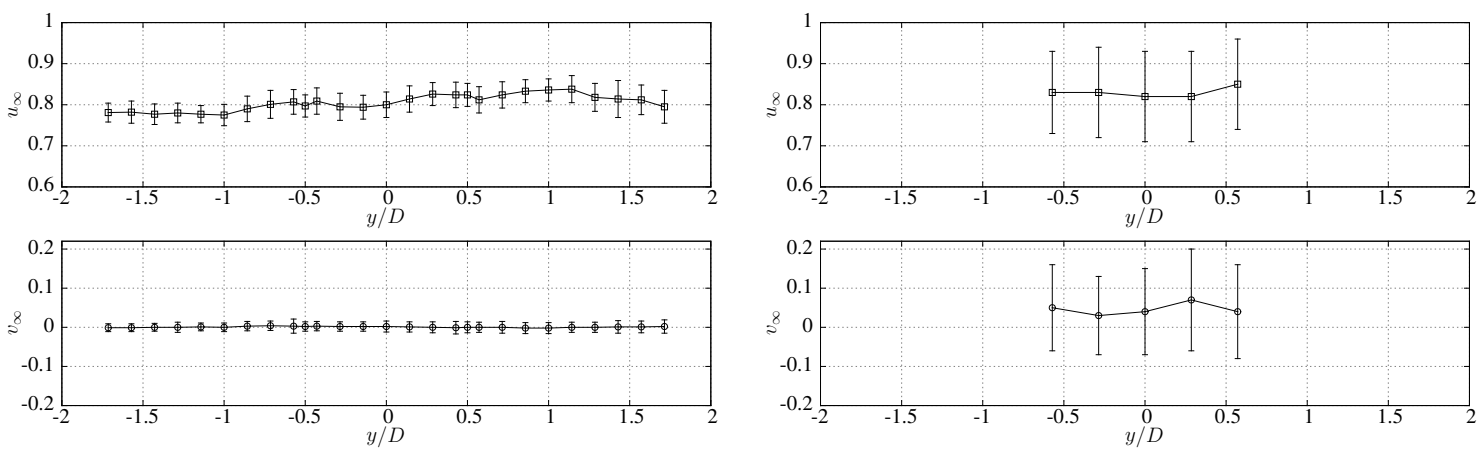

(a) $I_{\infty}=3 \%, U_{\infty}=0.8 \mathrm{~m} \cdot \mathrm{s}^{-1}$

(b) $I_{\infty}=15 \%, U_{\infty}=0.83 \mathrm{~m} \cdot \mathrm{s}^{-1}$

Figure 3: Upstream $u_{\infty}$ and $v_{\infty}$ velocity profiles across the tank, for $I_{\infty}=3 \%$ with $U_{\infty}=0.8 \mathrm{~m} \cdot \mathrm{s}^{-1}$ (left), and $I_{\infty}=15 \%$ with $U_{\infty}=0.83 \mathrm{~m} \cdot \mathrm{s}^{-1}$ (right). Symbols $\square$ (resp. o) represent the mean value $\bar{u}_{\infty}\left(\right.$ resp. $\left.\bar{v}_{\infty}\right)$, while error bars represent the corresponding standard deviation $\sigma\left(u_{\infty}\right)$ (resp. $\left.\sigma\left(v_{\infty}\right)\right)$.

\subsection{Turbine model description}

The model consists of a three-bladed horizontal axis turbine, which is $D=2 R=0.7 \mathrm{~m}$ in diameter. The rotor is connected to a motor-gearbox assembly consisting of a gearbox, a DC motor, a ballast load and a motor speed control unit [25], providing an active rotor speed control. The turbine blades are designed from a NACA 63418 profile. A more detailed profile description is given in Table 3.

The blockage ratio $\alpha$ is defined as the ratio between the rotor cross-section area $S=\pi R^{2}$ and the flume tank transverse area $A=W \cdot H$ :

$$
\alpha=\frac{S}{A}=\frac{\pi R^{2}}{W \cdot H}
$$

where $W=4 \mathrm{~m}$ and $H=2 \mathrm{~m}$ respectively denote the flume tank width and depth. In our study, the blockage ratio is then around $\alpha \approx 4.8 \%$.

The Tip Speed Ratio (TSR) is classically defined as the ratio between the tip velocity and the upstream flow velocity as follows:

$$
\mathrm{TSR}=\frac{|\boldsymbol{\Omega}| R}{U_{\infty}}=\frac{\left|\Omega_{x}\right| R}{U_{\infty}},
$$

where $\Omega$ is the rotor angular velocity and $\Omega_{x}$ is thus the axial rotation speed, $R$ is the rotor radius and $U_{\infty}$ is the upstream flow velocity. In our study, the turbine TSR varies from 0 to 10 . 


\begin{tabular}{cccc}
\hline$r / R$ & $c / R$ & Pitch $(\mathrm{deg})$ & $t / c(\%)$ \\
\hline 0.1333 & 0.0567 & 29.5672 & 80.0 \\
0.1500 & 0.0567 & 29.5672 & 100.0 \\
0.1550 & 0.0567 & 29.5672 & 100.0 \\
\hline 0.1983 & 0.1521 & 25.6273 & 36.0 \\
0.2417 & 0.2474 & 22.1491 & 21.3 \\
0.2850 & 0.2375 & 19.3031 & 21.4 \\
0.3283 & 0.2259 & 16.9737 & 21.7 \\
0.3717 & 0.2141 & 15.0538 & 22.0 \\
0.4150 & 0.2029 & 13.4572 & 22.2 \\
0.4583 & 0.1925 & 12.1169 & 22.4 \\
0.5017 & 0.1829 & 10.9815 & 22.5 \\
0.5450 & 0.1743 & 10.0114 & 22.5 \\
0.5883 & 0.1665 & 9.1761 & 22.4 \\
0.6317 & 0.1594 & 8.4516 & 22.2 \\
0.6750 & 0.1529 & 7.8191 & 21.9 \\
0.7183 & 0.1471 & 7.2638 & 21.5 \\
0.7617 & 0.1418 & 6.7735 & 20.9 \\
0.8050 & 0.1370 & 6.3387 & 20.2 \\
0.8483 & 0.1325 & 5.9514 & 19.5 \\
0.8917 & 0.1285 & 5.6050 & 18.6 \\
0.9350 & 0.1247 & 5.2941 & 18.0 \\
0.9783 & 0.1213 & 5.0143 & 18.0 \\
1.0000 & 0.0655 & 4.8743 & 25.0 \\
\hline
\end{tabular}

Table 3: Detailed blade profile description.

Finally, the radius-based Reynolds number is given by:

$$
\operatorname{Re}_{\infty}=\frac{U_{\infty} R}{\nu}
$$

where $\nu$ denotes the fluid kinematic viscosity and is approximately $\nu \approx 10^{-6} \mathrm{~m}^{2} \cdot \mathrm{s}^{-1}$. The studied Reynolds number range is then directly deduced from the $U_{\infty}$ range mentioned above, which gives $\operatorname{Re}_{\infty} \in[140,000 ; 420,000]$. The whole structure description summary is presented in Table 4a and a picture is given in Figure $4 \mathrm{~b}$.

\subsection{Force and moment measurements}

The force acting on the structure is obtained by means of a six-components load cell, which measures the three force components and the three moment components, at a $100 \mathrm{~Hz}$ acquisition frequency. A torque sensor, directly fixed between the rotor and the motor, provides a more accurate measurement of the axial torque than the one given by the load cell, at a $100 \mathrm{~Hz}$ acquisition frequency as well.

The power coefficient $C_{P}$ is defined as the proportion of power $\mathcal{P}$ retrieved by the turbine as compared to the maximum available power $\mathcal{P}_{\infty}$ from the incoming flow through the rotor area $S$ :

$$
C_{P}=\frac{\mathcal{P}}{\mathcal{P}_{\infty}}=\frac{\mathcal{M}_{x} \Omega_{x}}{\frac{1}{2} \rho S U_{\infty}^{3}}=\frac{\mathcal{M}_{x} \Omega_{x}}{\frac{1}{2} \rho \pi R^{2} U_{\infty}^{3}},
$$




\begin{tabular}{ll}
\hline Description & IFREMER-LOMC \\
\hline Profile & NACA 63418 \\
Rotor Radius $(R)$ & $350 \mathrm{~mm}$ \\
Hub Radius & $46 \mathrm{~mm}$ \\
Hub length & $720 \mathrm{~mm}$ \\
Pitch (set angle) & $0^{\circ}$ \\
Studied TSR & {$[0-10]$} \\
Sense of rotation & counter-clockwise \\
Reynolds $\left(\mathrm{Re}_{\infty}\right)$ & {$[140-420] \cdot 10^{3}$} \\
\hline
\end{tabular}

(a) Turbine model general description.

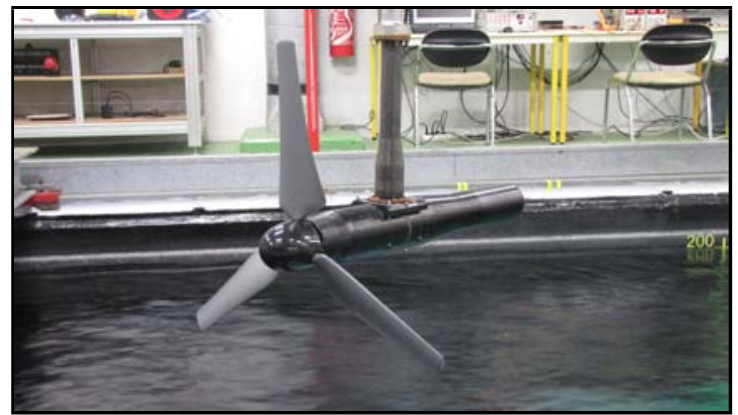

(b) Photography of a turbine model

Figure 4: Turbine models description.

where $\rho$ is the fluid density, $S=\pi R^{2}$ is the turbine cross-section area and $\mathcal{M}_{x}$ is the axial moment - or torque, defined as the $x$-component moment. Similarly, the thrust coefficient $C_{T}$ is defined as the axial force $\mathcal{F}_{x}$ acting upon the turbine as compared to the kinetic energy of the incoming flow through $S$ :

$$
C_{T}=\frac{\mathcal{F}_{x}}{\frac{1}{2} \rho \pi R^{2} U_{\infty}^{2}},
$$

The $\mathcal{F}_{x}$ measured here actually includes the axial force on the whole structure, that is the blades, the hub and the mast. Obviously, the $\mathcal{M}_{x}$ and $\mathcal{F}_{x}$ values used to compute those coefficients are time-averaged values. The measurement (and thus the averaging) duration is $T=100$ seconds, which provides converged values of $C_{P}$ and $C_{T}$ (cf. Appendix C).

\section{6. $L D V$ measurements}

The flow velocity measurements are performed by means of a Laser Doppler Velocimetry (LDV) system described in $[21,26]$. The LDV measurements are performed on a grid whose nodes $\left(X_{i}, Y_{i}\right)$ are arranged as follows:

- $X_{1}=1.2 D$ and $X_{i}=i \times D$ for $i=2, \ldots, 10$;

- $Y_{i}=-1.2+(i-1) \times 0.1 \mathrm{~m}$ for $i=1, \ldots, 25$, with two additional positions $Y_{26}=-Y_{27}=R=$ $0.35 \mathrm{~m}$.

The laser used for the measurements is described in Table 4.

\begin{tabular}{ll}
\hline Description & DANTEC FiberFlow \\
\hline$x$-wavelength & $488 \mathrm{~nm}$ \\
$y$-wavelength & $514.5 \mathrm{~nm}$ \\
Focal length (in water) & $500 \mathrm{~mm}$ \\
Measurement area $\delta x \times \delta y$ & $0.1 \times 2.5 \mathrm{~mm}^{2}$ \\
\hline
\end{tabular}

Table 4: Description of the DANTEC FiberFlow laser used for LDV measurements. 
The discrete time-averaged velocity, corresponding to the discrete version of equation (4), is basically computed as follows:

$$
\overline{\mathbf{u}}(\mathbf{x}) \approx \frac{1}{N} \sum_{k=1}^{N} \mathbf{u}\left(\mathbf{x}, t_{k}\right)
$$

where $t_{k}$ denotes the measurement instants and $N$ denotes the total number of measurements during the averaging period $[0 ; T]$. As a consequence, the Reynolds stress terms are approximated by:

$$
R_{i j} \approx \frac{1}{N} \sum_{k=1}^{N}\left[u_{i}\left(\mathbf{x}, t_{k}\right)-\bar{u}_{i}(\mathbf{x})\right]\left[u_{j}\left(\mathbf{x}, t_{k}\right)-\bar{u}_{j}(\mathbf{x})\right],
$$

with each $\bar{u}_{i}(\mathbf{x})$ approximated thanks to equation (13).

The measurement duration on each node is $T=100$ seconds. This duration is justified by the convergence graphs presented in Appendix B on various measured quantities, both for $I_{\infty}=3 \%$ (Fig. B.15) and $I_{\infty}=15 \%$ (Fig. B.16). The observed data rate is between 6 and 33Hz. Axial velocity fluctuation spectra, for upstream conditions and at one point behind the turbine, are given in Appendix D.

\subsection{Downstream flow characterisation}

The downstream turbulence intensity rate in the $x O y$ plane at given location $(x, y, z)$ is evaluated as follows:

$$
I(x, y, z)=100 \sqrt{\frac{\frac{1}{2}\left[\sigma^{2}(u)+\sigma^{2}(v)\right]}{\bar{u}^{2}+\bar{v}^{2}}} .
$$

Since it corresponds to a $2 D$ turbulence intensity, it should thus be compared to the appropriate $I_{\infty}^{2 D}$ upstream intensity, as explained previously in section 2.3. Likewise, the $2 D$ dimension-free downstream Reynolds shear stress intensity is defined by:

$$
{\overline{u^{\prime} v^{\prime}}}^{*}(x, y, z)=\sqrt{\frac{\left|\overline{u^{\prime} v^{\prime}}\right|}{\bar{u}^{2}+\bar{v}^{2}}}
$$

where the $*$ superscript indicates that this quantity represents a dimension-free version of the Reynolds shear stress $\overline{u^{\prime} v^{\prime}}$ (see paragraph 2.9).

\subsection{Integrated velocity and turbulence intensity on a disc}

A commonly used way of evaluating the velocity deficit is to consider the centreline velocity deficit, that is to say the velocity deficit at punctual locations along the turbine axis, defined at a given distance $x$ from the turbine as follows:

$$
\bar{u}_{0}(x)=\bar{u}(x, 0,0) .
$$

And thus, the centreline axial velocity deficit at given location $(x, 0,0)$, expressed as a percentage, is naturally defined as:

$$
\gamma_{0}(x)=100\left(1-\frac{\bar{u}_{0}(x)}{U_{\infty}}\right) .
$$




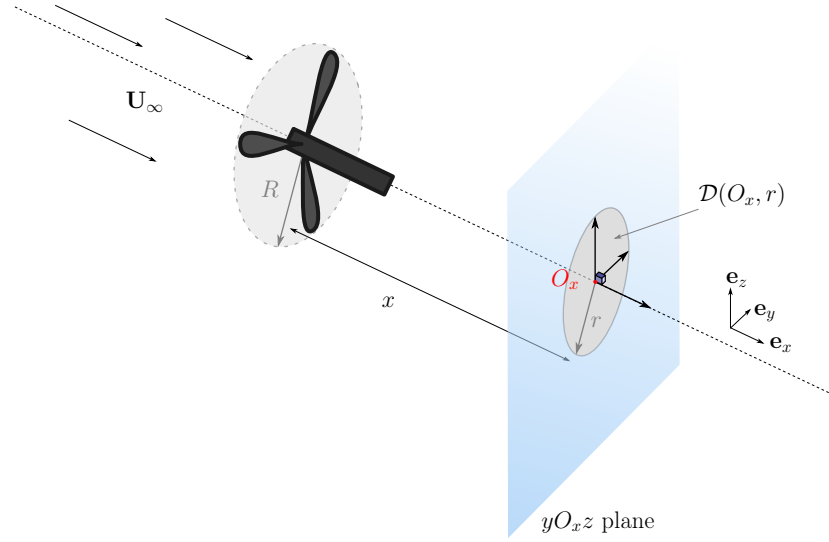

(a) Illustration of the integration $\operatorname{disc} \mathcal{D}\left(O_{x}, r\right)$ in the $y O_{x} z$ plane. The disc radius $r$ (i.e. the integration radius) is not necessarily equal to the rotor radius $R$.

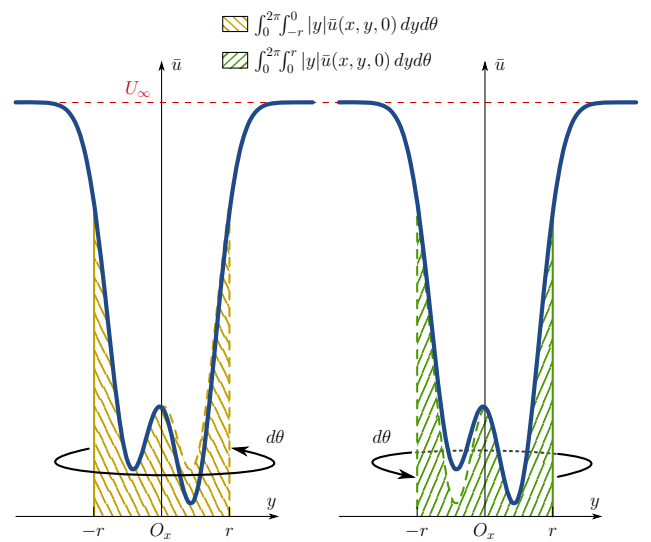

(b) Illustration of the pseudo-axisymmetric axial velocity integration on a disc. On the left (yellow), the integration is performed on the left half of the profile. Conversely, on the right (green), the integration is performed on the right half of the profile.

Figure 5: Illustration of the disc-integrated average at a given axial distance $x$ from the turbine, i.e. at location $O_{x}(x, 0,0) . O_{x}$ may represent the potential location of a downstream turbine of radius $r$, and thus $\mathcal{D}\left(O_{x}, r\right)$ would represent its cross-section area.

It is important to note that in the case of radial gradient in the velocity profiles, the centreline velocity deficit may not be a relevant indicator of the actual velocity deficit in the whole turbine's area of influence. In order to further evaluate the velocity deficit as it would be perceived by a potential downstream turbine, a velocity deficit integrated on a disc is defined at a point $O_{x}(x, 0,0)$ located at a given distance $x$ from the upstream turbine. The disc is parallel to the cross-sectional area of the turbine, that is to say it is defined in the $y O_{x} z$ plane. Figure $5 \mathrm{a}$ depicts such a disc at some arbitrary location $O_{x}$ and with some arbitrary radius $r$, whose choice will be discussed later on. The disc-integrated velocity (from which is computed the disc-integrated deficit) is then obtained by computing the integral in an ad hoc polar coordinate system.

In the case of axi-symmetric velocity profiles, the disc integral would reduce to a one-dimensional integral depending on the radial coordinate only. This radial coordinate is naturally set to be $|y|$. However, as the velocity profile is not exactly axi-symmetric [21, 22], the final integrated value is obtained by taking the mean of those two integrals. The integration is performed successively for positive and negative values of $y$, that is to say on $[0 ; r]$ and $[-r ; 0]$. This integration process is illustrated on Figure 5b.

Finally, the resulting disc-integrated mean velocity is normalised by the disc-integrated value of $U_{\infty}$ in order to make it dimension-free. Thus, the mean axial velocity at a given location $O_{x}(x, 0,0)$, integrated on a disc $\mathcal{D}\left(O_{x}, r\right)$ of centre $O_{x}$ and radius $r$ defined in the $y O_{x} z$ plane is eventually 
given by:

$$
\begin{aligned}
\hat{\bar{u}}_{r}^{*}(x) & =\frac{\iint_{\mathcal{D}\left(O_{x}, r\right)} \bar{u}(x, y, z) d y d z}{\iint_{\mathcal{D}\left(O_{x}, r\right)} U_{\infty} d y d z}=\frac{\iint_{\mathcal{D}\left(O_{x}, r\right)} \bar{u}(x, y, z) d y d z}{U_{\infty} \iint_{\mathcal{D}\left(O_{x}, r\right)} d y d z}=\frac{\hat{\bar{u}}_{r}(x)}{U_{\infty}} \\
& =\frac{\frac{1}{2}\left[\int_{0}^{2 \pi} \int_{0}^{r}|y| \bar{u}(x, y, 0) d y d \theta+\int_{0}^{2 \pi} \int_{-r}^{0}|y| \bar{u}(x, y, 0) d y d \theta\right]}{\pi r^{2} U_{\infty}} \\
& \simeq \frac{1}{r^{2} U_{\infty}} \int_{-r}^{r}|y| \bar{u}(x, y, 0) d y,
\end{aligned}
$$

where $\hat{\bar{u}}_{r}=\frac{\iint_{\mathcal{D}\left(O_{x}, r\right)} \bar{u}(x, y, z) d y d z}{\iint_{\mathcal{D}\left(O_{x}, r\right)} d y d z}$ represents a normalised disc-integrated velocity.

The mean axial velocity deficit at a given location $O_{x}(x, 0,0)$, integrated on $\mathcal{D}\left(O_{x} ; r\right)$, expressed as a percentage, is then basically given by:

$$
\hat{\gamma}_{r}(x)=100\left(\frac{U_{\infty}-\hat{\bar{u}}_{r}(x)}{U_{\infty}}\right)=100\left(1-\frac{\hat{\bar{u}}_{r}(x)}{U_{\infty}}\right)=100\left(1-\hat{\bar{u}}_{r}^{*}(x)\right) .
$$

The same disc-integration process can be carried out on the turbulence intensity. The mean turbulence intensity rate at given location $O_{x}(x, 0,0)$, integrated on $\mathcal{D}\left(O_{x} ; r\right)$, is then given by:

$$
\hat{I}_{r}(x)=\frac{\iint_{\mathcal{D}\left(O_{x}, r\right)} I(x, y, z) d y d z}{\iint_{\mathcal{D}\left(O_{x}, r\right)} d y d z} \simeq \frac{1}{r^{2}} \int_{-r}^{r}|y| I(x, y, 0) d y,
$$

while the commonly used centreline turbulence intensity rate at given location $O_{x}(x, 0,0)$ is basically given by:

$$
I_{0}(x)=I(x, 0,0)
$$

\subsection{Dimension-free quantities}

Every presented quantity is made dimension-free by dividing it by a characteristic homogeneous quantity. Most of these dimension-free quantities have just been presented in this section. Besides, any length is made dimension-free by means of the rotor diameter $D$, and any flow velocity is made dimension-free by means of the upstream axial velocity $U_{\infty}$.

Moreover, the dimension-free version of any dimensional quantity $q$ is denoted by $q^{*}$. However, some quantities are directly presented dimension-free, because they do not have any dimensional version or because their dimensional version is irrelevant. This is the case, for example, of $I, \gamma_{0}$, $\hat{\gamma}_{r}$. Other specific dimension-free quantities such as the Tip Speed Ratio (TSR) or the thrust and power coefficients $\left(C_{P}\right.$ and $\left.C_{T}\right)$ are, of course, denoted as in the literature. Table 5 is a summary of the dimension-free quantities and their corresponding dimensional versions.

\section{Performance evaluation}

Several studies have being carried out on the evaluation of marine current turbines performances $[13,21]$. The present study really aims at giving complete and detailed data sets of both turbine performances (i.e. power and thrust coefficients) and wake flow characterisation (section 4). A previous study [21] was presented in a similar manner but the turbine blade profiles were confidential. As a consequence, all the results were made dimension-free by some unknown coefficient, 


\begin{tabular}{cll}
\hline dimensional & dimension-free & comment \\
\hline$x$ & $x^{*}=x / D$ & \\
$y$ & $y^{*}=y / D$ & \\
\hline $\bar{u}$ & $\bar{u}^{*}=\bar{u} / U_{\infty}$ & \\
$\hat{\bar{u}}_{r}$ & $\hat{\bar{u}}_{r}^{*}=\hat{\bar{u}}_{r} / U_{\infty}$ & \\
\hline- & $\gamma_{0}(x)=100\left(1-\bar{u}^{*}(x, 0,0)\right)$ & eq. (18) \\
- & $\hat{\gamma}_{r}=100\left(1-\hat{\bar{u}}_{r}^{*}\right)$ & eq. (22) \\
\hline- & $I_{\infty}, I_{\infty}^{2 D}, I(x, y, z), \hat{I}_{r}(x), I_{0}(x)$ & eq. (6), (7), (15), (23), (24) \\
\hline $\bar{u}^{\prime} v^{\prime}$ & $\bar{u}^{\prime} v^{*}=\sqrt{\left|\bar{u}^{\prime} v^{\prime}\right| /\left(\bar{u}^{2}+\bar{v}^{2}\right)}$ & eq. (16) \\
\hline- & $\mathrm{TSR}^{*}=|\Omega| R / U_{\infty}$ & eq. (9) \\
- & $C_{P}=\mathcal{M} x \Omega_{x} /\left(\frac{1}{2} \rho \pi R^{2} U_{\infty}^{3}\right)$ & eq. (11) \\
- & $C_{T}=\mathcal{F}_{x} /\left(\frac{1}{2} \rho \pi R^{2} U_{\infty}^{2}\right)$ & eq. (12) \\
\hline
\end{tabular}

Table 5: Dimension-free quantities.

which makes comparisons with new studies impossible. Another significant difference stems in the torque measurement, which is now performed with a torque sensor rather than the load cell, so as to obtain more accurate and less noisy measurements (see Appendix C). The main goal here is to provide the scientific community with relevant data sets for comparison with future studies. Additionally, $C_{P}$ and $C_{T}$ representative spectra are shown in Appendix D. More detailed spectral analyses were carried out on similar configurations by Maganga et al. [21] and Gaurier et al. [27].

\subsection{Power coefficient}

Figures $6 \mathrm{a}$ and $6 \mathrm{~b}$ depict the performance curves for different upstream velocities $U_{\infty}$ and the two studied ambient turbulence intensities, namely $I_{\infty}=3 \%$ and $I_{\infty}=15 \%$. Except for $U_{\infty}=0.4 \mathrm{~m} \cdot \mathrm{s}^{-1}$ with the lower ambient turbulence intensity, the turbine operates properly and the $C_{P}$ curves are very similar to those presented in the literature [13, 21]. For both ambient turbulence intensities, it is striking to observe that the turbine operating range is always contained in $3 \leqslant$ TSR $\leqslant 6$. For $I_{\infty}=3 \%$ and except for $U_{\infty}=0.4 \mathrm{~m} \cdot \mathrm{s}^{-1}$, the $C_{P}$ values are always slightly above 0.4 (Fig. 6a), whereas they are slightly below this value for almost every upstream velocity at $I_{\infty}=15 \%$ (Fig. 6b). However, except for $U_{\infty}=0.4 \mathrm{~m} \cdot \mathrm{s}^{-1}$ again, the $C_{P}$ curves tend to collapse into a single curve for $I_{\infty}=3 \%$ all over the TSR range, while they tend to differ for $I_{\infty}=15 \%$. With this ambient turbulence intensity, the higher the upstream velocity $U_{\infty}$, the higher the $C_{P}$ curve. Indeed, the maximum $C_{P}$ for $U_{\infty}=0.83$ or $0.62 \mathrm{~m} \cdot \mathrm{s}^{-1}$ is almost $10 \%$ lower than for $U_{\infty}=1.03 \mathrm{~m} \cdot \mathrm{s}^{-1}$. In addition, difference between the curves increase with the TSR.

\subsection{Thrust coefficient}

The thrust evolution of the considered turbine also leads to a similar conclusion: the turbine behaviour is weakly dependent on the ambient turbulent intensity $I_{\infty}$. In fact, all the $C_{T}$ curves 


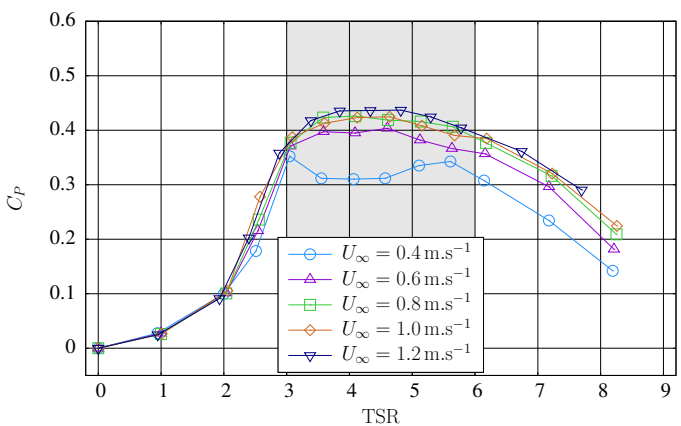

(a) $I_{\infty}=3 \%$

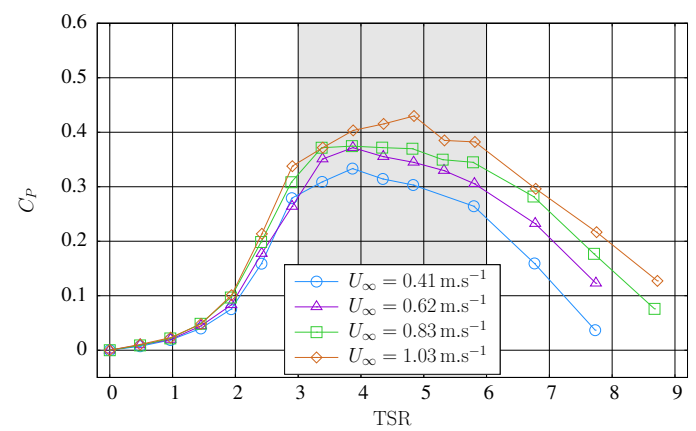

(b) $I_{\infty}=15 \%$

Figure 6: Evaluation of the power coefficient $C_{P}$ function of the TSR, for $I_{\infty}=3 \%$ (left) and $I_{\infty}=15 \%$ (right).

almost superimpose for $I_{\infty}=3 \%$ (Fig. 7a), except once more for $U_{\infty}=0.4 \mathrm{~m} \cdot \mathrm{s}^{-1}$, as for the $C_{P}$ curves. In the operating range $(3 \leqslant \mathrm{TSR} \leqslant 6)$, the $C_{T}$ values increase from approximately 0.7 to plateau of about 0.80 or 0.85 from TSR $\approx 4$ even until the last TSR value (TSR $\approx 8$ ). A similar analysis can be performed for $I_{\infty}=15 \%$ (Fig. $7 \mathrm{~b}$ ), the two main differences being that the $C_{T}$ curves all superimpose without exception (from $U_{\infty}=0.41 \mathrm{~m} \cdot \mathrm{s}^{-1}$ to $1.03 \mathrm{~m} \cdot \mathrm{s}^{-1}$ ) and that there is no distinct plateau. In the operating range, the curves increase from $C_{T} \approx 0.7$ to a maximum value of 0.8 approximately at TSR $\approx 6$, and then decreases.

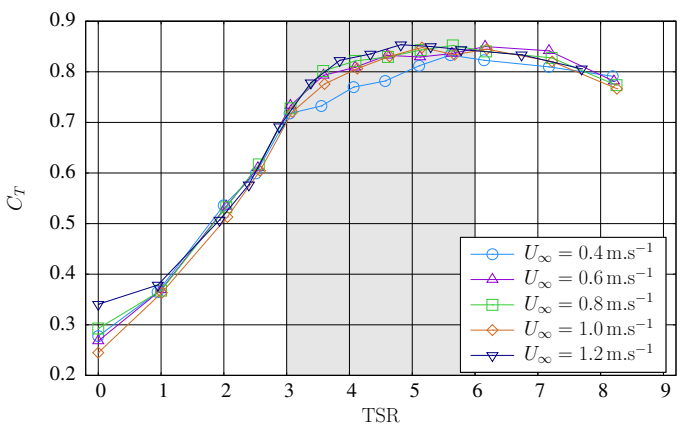

(a) $I_{\infty}=3 \%$

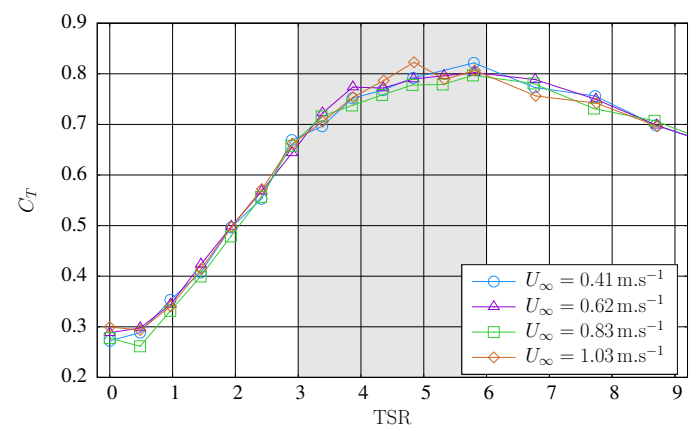

(b) $I_{\infty}=15 \%$

Figure 7: Evaluation of the thrust $C_{T}$ coefficient function of the TSR, for $I_{\infty}=3 \%$ (left) and $I_{\infty}=15 \%$ (right).

\subsection{Standard deviation}

Nevertheless, the ambient turbulence intensity $I_{\infty}$ does have a significant influence, not on the mean values of $C_{P}$ and $C_{T}$, but on their standard deviation values $\left(\sigma_{C_{P}}\right.$ and $\left.\sigma_{C_{T}}\right)$ depicted on Figures $8 \mathrm{a}$ and $8 \mathrm{~b}$. Indeed, whatever the $C_{P}$ or $C_{T}$ in the operating range, $\sigma_{C_{P}}$ and $\sigma_{C_{T}}$ are always somehow 2.5 times higher with $I_{\infty}=15 \%$ than with $I_{\infty}=3 \%$. The observation is still valid outside the operating range in the upper part of the TSR values (i.e. TSR $\geqslant 6$ ). However, for lower values (i.e. TSR $\leqslant 3$ ), $\sigma_{C_{P}}$ tends to collapse into a single curve whatever the ambient turbulence intensity $I_{\infty}$, whereas $\sigma_{C_{T}}$ remains almost twice higher with $I_{\infty}=15 \%$. The described 
behaviour is valid whatever the upstream velocity value except for $U_{\infty}=0.4 \mathrm{~m} \cdot \mathrm{s}^{-1}$ with $I_{\infty}=3 \%$ as discussed earlier. The fact that $\sigma_{C_{P}}$ and $\sigma_{C_{T}}$ are always 2.5 times higher with $I_{\infty}=15 \%$ than with $I_{\infty}=3 \%$ is far from being negligible.

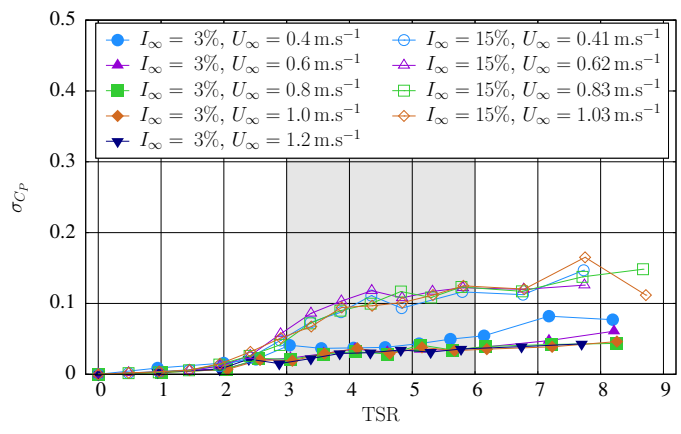

(a) $\sigma_{C_{P}}$

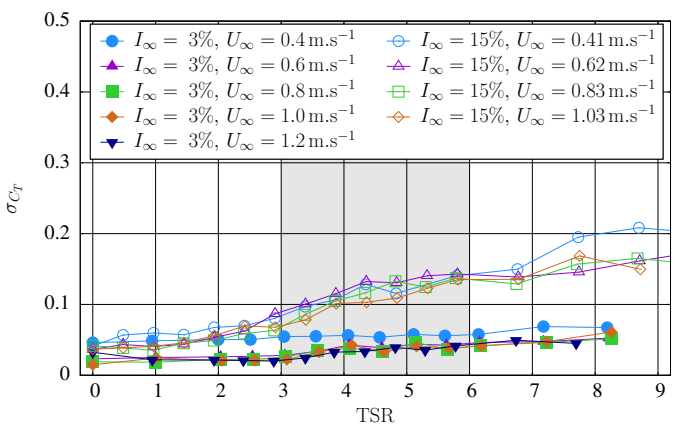

(b) $\sigma_{C_{T}}$

Figure 8: Standard deviation of the power $C_{P}$ (left) and thrust $C_{T}$ (right) coefficients, for $I_{\infty}=3 \%$ and $15 \%$.

\subsection{Discussion}

In terms of industrial applications, the relatively similar power and thrust behaviour of the studied turbine is an interesting feature because it enables its installation whatever the environmental ambient turbulence. This observation is also appropriate if the turbulence intensity varies with time at a precise location, either through the action of the tide or owing to the weather conditions. This turbine geometry (blade profile, length, chord and twist) makes it very robust to ambient upstream velocity conditions, which represents an important issue in the event of interaction effects between turbines. As a matter of fact, in a turbine wake, the turbulence intensity increases. However, medium to small scales coherent structures appear and add to the initially homogeneous turbulence. Consequently, the downstream turbine does not behave as if it were immersed in a flow with a given ambient turbulence intensity. This issue will be addressed in the sequel of the present paper, see Part II: two interacting turbines [1].

As concerns the fatigue of blades [20,27], as well as gear boxes and electrical generators, standard deviation of both power and thrust coefficients may have a major impact especially on the design and hence, on the global cost of the machine.

The ambient turbulence intensity thus influences the machine behaviour, in terms of maximum retrieved power to a small extent, but most importantly and to a larger extent, in terms of force and torque fluctuations.

\section{Wake characterisation}

\subsection{Velocity, turbulence intensity and Reynolds shear stress maps}

Figure 9 presents mean axial velocity, turbulence intensity rate and Reynolds shear stress maps, from $1.2 D$ to $10 D$ downstream of the turbine. The upstream velocity is $0.8 \mathrm{~m} \cdot \mathrm{s}^{-1}$ and the turbine TSR is 3.67. As previously, two upstream turbulence intensity rates are considered and compared, namely $I_{\infty}=3 \%$ and $I_{\infty}=15 \%$. In order to make our experimental data available in a more 


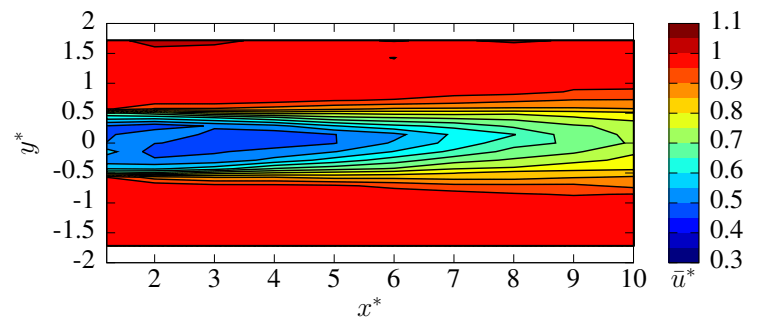

(a) Axial velocity map $\left(I_{\infty}=3 \%\right)$

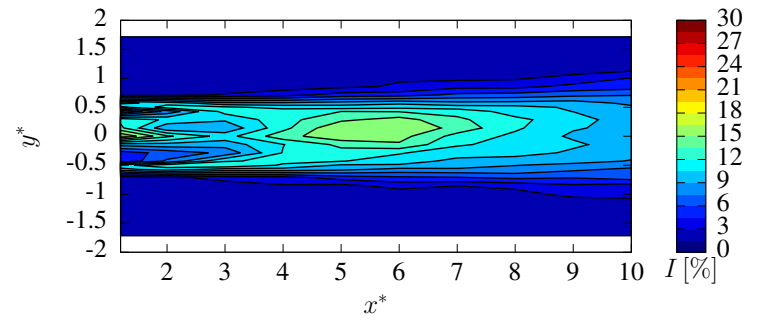

(c) Turbulence intensity map $\left(I_{\infty}=3 \%\right)$

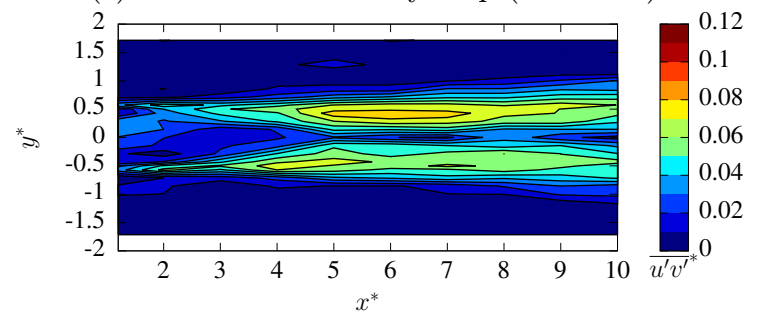

(e) Reynolds shear stress map $\left(I_{\infty}=3 \%\right)$

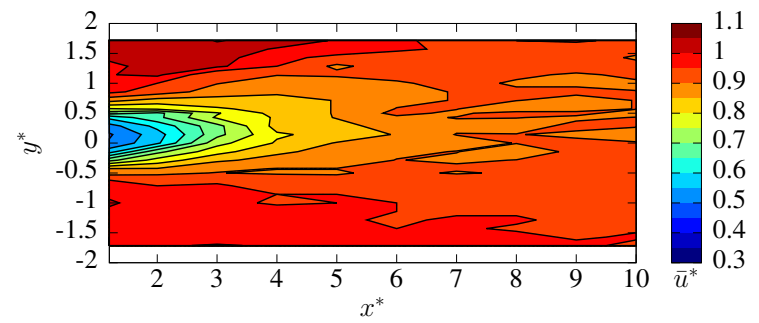

(b) Axial velocity map $\left(I_{\infty}=15 \%\right)$

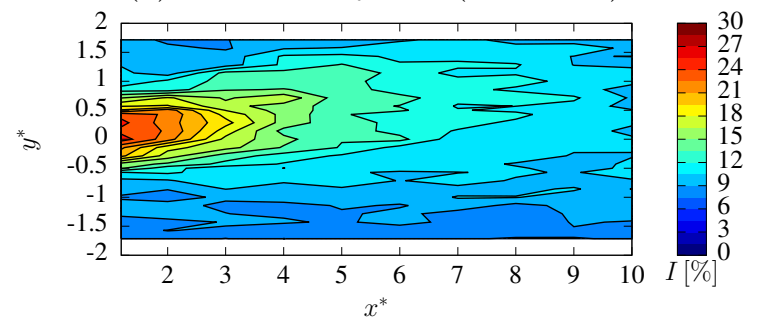

(d) Turbulence intensity map $\left(I_{\infty}=15 \%\right)$

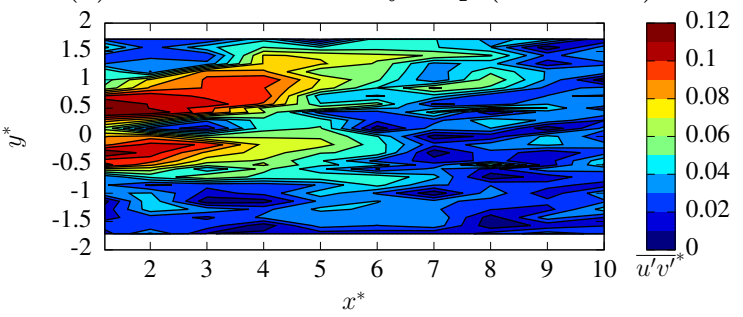

(f) Reynolds shear stress map $\left(I_{\infty}=15 \%\right)$

Figure 9: Wake behind a turbine with TSR $=3.67, U_{\infty}=0.8 \mathrm{~m} \cdot \mathrm{s}^{-1}$ and for $I_{\infty}=3 \%$ (left) and $I_{\infty}=15 \%$ (right).

convenient way, velocity and turbulence intensity profiles behind the turbine are provided in Appendix A.

The first striking observation is that the wake effects decrease much faster with a higher upstream turbulence level, in terms of both axial velocity deficit and downstream turbulence intensity rate. As a matter of fact, while with $I_{\infty}=3 \%$, the velocity deficit remains well pronounced even 10 diameters downstream (cf. Figure 9a), with $I_{\infty}=15 \%$, the flow recovers most of its velocity from 6 diameters (cf. Figure 9b).

The same observation holds for the downstream turbulence intensity rate. With $I_{\infty}=3 \%$, the flow does not recover its turbulence upstream conditions even after 10 diameters, where the turbulence intensity rate remains much higher than $3 \%$, with approximately $9 \%$. It is striking to realise that the turbulence intensity peak is not located immediately behind the turbine, but between 5 and 7 diameters downstream. This is consistent with previous observations made by [17, 21], for instance. Looking at the velocity profiles (Fig. A.12 in Appendix A), one can observe that this high turbulence intensity zone exactly corresponds to a modification in the velocity profiles shape, from a top hat to a bell shape velocity profile. This kind of shape transition has already been identified in a previous study [21] with a different turbine configuration, in a flow with a turbulence intensity 
rate of $5 \%$. This shape modification in the velocity profiles is linked to the merging of the mixing layers. On Figure 9e, the merging of the two mixing layers can clearly be identified between $x^{*}=5$ and 7. On the contrary, with $I_{\infty}=15 \%$, the highest turbulence intensity zone is observed in the near wake of the turbine $\left(0 \leqslant x^{*} \leqslant 3\right)$ and rapidly decreases. In addition, the upstream conditions are fully recovered 10 diameters downstream.

Another observation that can be drawn from Figure 9 is that the wake shape is larger and more diffuse with $I_{\infty}=15 \%$ than it is with $I_{\infty}=3 \%$. On the one hand, with $I_{\infty}=3 \%$, both axial velocity and turbulence intensity are only disturbed in a limited axial strip, which is approximately one diameter large and axially aligned with the turbine. This strip slightly enlarges along with flow direction to become between 1.5 to 2 diameters large at 10 diameters downstream of the turbine. On the other hand, with $I_{\infty}=15 \%$, the wake shape is less easily distinguishable. It rapidly spreads out in radial directions, unlike with $I_{\infty}=3 \%$. These last observations are clearly related to the Reynolds shear stress maps depicted on Figures 9e and 9f. From Figure 9e $\left(I_{\infty}=3 \%\right)$, two zones can easily be distinguished. For $0 \leqslant x^{*} \leqslant 5$, the shear layers start very thin just behind the turbine and grow thicker. Indeed, from $x^{*} \geqslant 5$, the shear layers are much larger. The first zone $\left(0 \leqslant x^{*} \leqslant 5\right)$ corresponds to sharp velocity gradients (cf. Figures 10 and A.12a in Appendix A). The presence of blade tip vortices was also observed for a similar configuration [21].

Since the upstream flow has a low ambient turbulence intensity rate, $I_{\infty}=3 \%$, the shear layers naturally develop without being too much perturbed by the upstream turbulence. From $x^{*} \geqslant 5$, the shear layers have completely developed and start to merge. This merging zone $5 \leqslant x^{*} \leqslant 7$ clearly corresponds to the highest turbulence intensity zone observed on Figure 9c. This last observation is another characteristic of the transition from a top hat to a bell shape velocity profile. Despite the differences in the turbine configuration (especially the blade design), its rotation speed and the ambient turbulence intensity, the wake behaviour with $I_{\infty}=3 \%$ is very similar to the one described in [21] with $I_{\infty}=5 \%$.

However, comparing the Reynolds shear stress values between Figure 9e and 9f clearly indicates that the flow morphology is somehow different with a higher turbulence intensity. The Reynolds shear stress maximum value is about 1.5 times higher with $I_{\infty}=15 \%$ than with $I_{\infty}=3 \%$, all other parameters equal. This indicates that the mixing is much more efficient with $I_{\infty}=15 \%$ than it is with the lower value.

As a matter of partial conclusion, the influence of ambient turbulence turns out to be significant. The higher the ambient turbulence $I_{\infty}$, the better the mixing and consequently, the shorter and weaker the wake influence.

\subsection{Disc-integrated velocity deficit and turbulence intensity}

Figure 10 illustrates the differences between the different evaluations of the velocity deficit in the near wake of a turbine (cf. section 2.8). For the disc-integrated velocity (eq. (21)), two values of $r$ are chosen. The first natural value is $R$, the turbine rotor radius. The second, $R^{+}$, slightly enlarges the integration area to the further two measured points along $y$. In other words, it enlarges the presumed wake area that would be perceived by a potential downstream turbine. Furthermore, together with $\bar{u}_{0}(x)$, those three values are useful to evaluate how homogeneous the flow is in this area. Indeed, if $\bar{u}_{0}(x)$ and $\hat{\bar{u}}_{R}(x)$ are similar, this means that the flow velocity is almost homogeneous on $\mathcal{D}\left(O_{x}, R\right)$. Conversely, if these values significantly differ, this indicates that high radial gradients are present in this area. The same remark holds for the difference between $\hat{\bar{u}}_{R}(x)$ and $\hat{\bar{u}}_{R^{+}}(x)$, except it indicates how homogeneous the flow is in the ring $\mathcal{D}\left(O_{x}, R^{+}\right)-\mathcal{D}\left(O_{x}, R\right)$. 


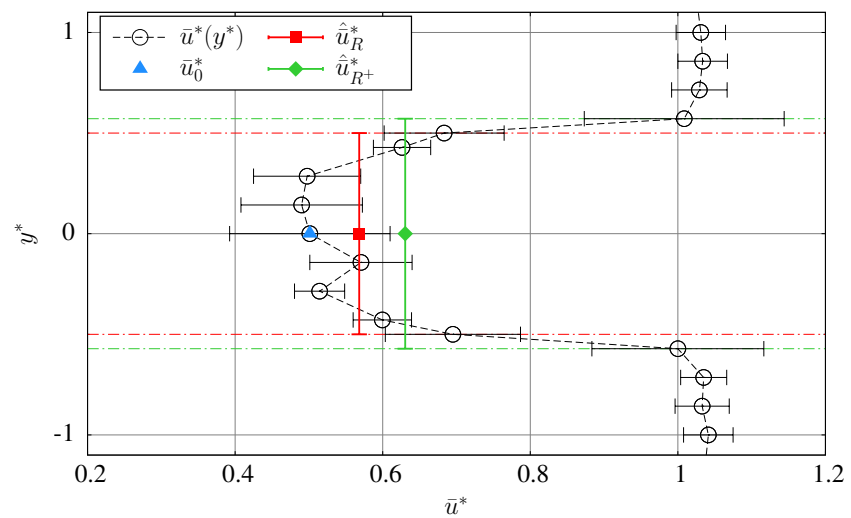

Figure 10: Mean axial velocity profile $\bar{u}^{*}\left(y^{*}\right)$ at $x^{*}=1.2$ with $U_{\infty}=0.8 \mathrm{~m} \cdot \mathrm{s}^{-1}, I_{\infty}=3 \%$ and TSR $=3.67$. The horizontal bars represent the axial velocity standard deviation $\sigma(u)$. The vertical bars indicate the mean axial velocity value averaged on a disc computed with equation (21).

The axial velocity profile depicted in Figure 10 is the first profile (issuing from Fig. 9a) measured in the wake of a turbine immersed in an incoming flow with a low turbulence intensity rate $I_{\infty}=3 \%$. The velocity profile $\bar{u}^{*}\left(y^{*}\right)$ clearly has a top hat shape. As discussed in section 2.8 , the velocity profile is not axi-symmetric. More precisely, the perturbation observed between $-0.5 \leqslant y^{*} \leqslant 0$ can be attributed to the interaction between the wake and the maintaining mast (see photography in Figure 4b or Figure 2). This asymmetry has also been observed for wind turbines [28]. In addition, the values of $\bar{u}_{0}(x), \hat{\bar{u}}_{R}(x)$ and $\hat{\bar{u}}_{R^{+}}(x)$ are rather different, as it was expected. If one wants to evaluate the power that would perceive a turbine situated in the wake, using the value of $\bar{u}_{0}(x)$ would clearly underestimate the potential power. Using the values $\hat{\bar{u}}_{R}(x)$ or $\hat{\bar{u}}_{R^{+}}(x)$ would improve the estimation but, owing to the sharp gradients at the shear layer location $\left(0.4 \leqslant\left|y^{*}\right| \leqslant 0.6\right)$, the difference between those two values is not negligible. The use of these three indicators of velocity would be of great interest, especially in the case of interactions that are discussed in Part II [1] of the present study. The difference between the centreline and the integrated deficits illustrates the heterogeneity of the flow at a given location.

Figure 11 translates Figures 9a to 9d in terms of centreline and disc-integrated velocity deficit (eq. (18) and (22)) and turbulence intensity rate (eq. (23) and (24)). A comparison of Figures 11a and $11 \mathrm{~b}$ provides valuable information. First, the three values of $\gamma_{0}, \hat{\gamma}_{R}$ or $\hat{\gamma}_{R^{+}}$never converge for an ambient turbulence intensity of $3 \%$. This behaviour was expected as the wake is well characterised even at 10 diameters behind the turbine. On the contrary, for $I_{\infty}=15 \%, \gamma_{0}, \hat{\gamma}_{R}$ and $\hat{\gamma}_{R^{+}}$ remain rather different only until $x^{*} \approx 3$ corresponding to the highest Reynolds shear stress area in Figure 9f. Then, $\hat{\gamma}_{R}$ and $\hat{\gamma}_{R^{+}}$almost superimpose from $x^{*} \approx 3$, whereas $\gamma_{0}$ still differs. Finally, the three indicators are very similar for $x^{*} \geqslant 5$, where the wake flow is hardly visible, which corresponds to the end of the wake (cf. Figure $9 \mathrm{~b}$ and $9 \mathrm{~d}$ ). As a matter of conclusion, these indicators provides precise information about the wake shape.

Another piece of information from these graphs is that the mean velocity deficit remains around $20 \%$ even 10 diameters behind the turbine for $I_{\infty}=3 \%$. This $20 \%$ velocity deficit at 10 diameters $\left(\bar{u}(10 D) \approx 0.8 U_{\infty}\right)$ actually corresponds to approximately $50 \%$ of available power deficit at this location $\left(p(10 D) \approx 0.5 \mathcal{P}_{\infty}\right)$. Indeed, as mentioned for the definition of the power coefficient (11), 


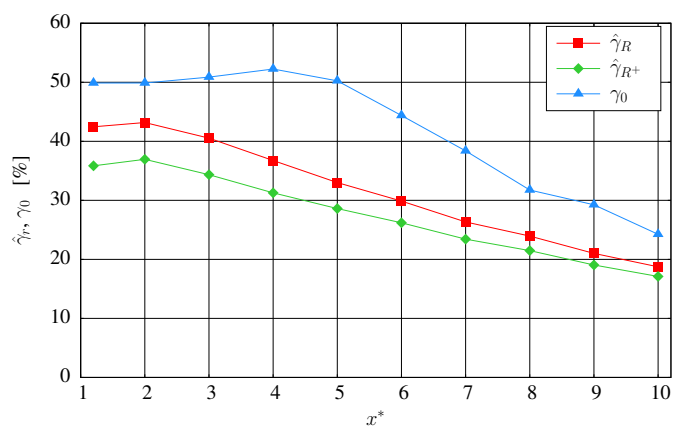

(a) Axial velocity deficit $\left(I_{\infty}=3 \%\right)$

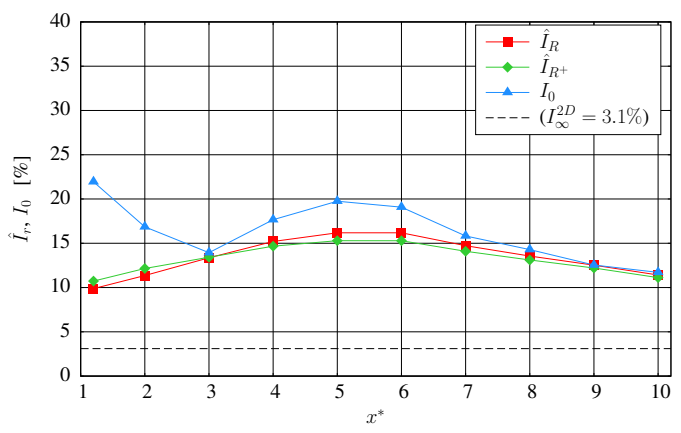

(c) Turbulence intensity $\left(I_{\infty}=3 \%, I_{\infty}^{2 D} \approx 3.1 \%\right)$

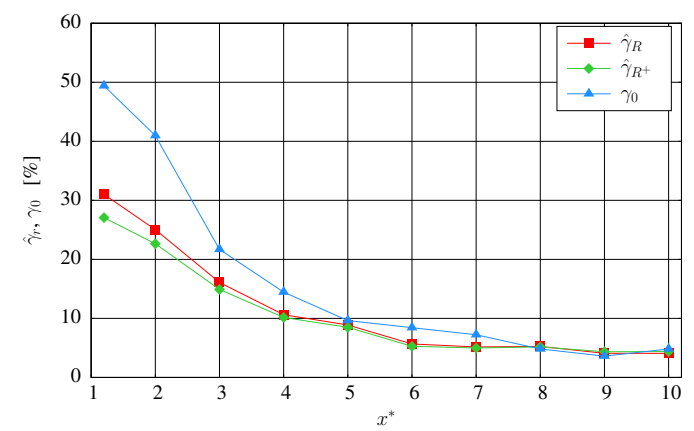

(b) Axial velocity deficit $\left(I_{\infty}=15 \%\right)$

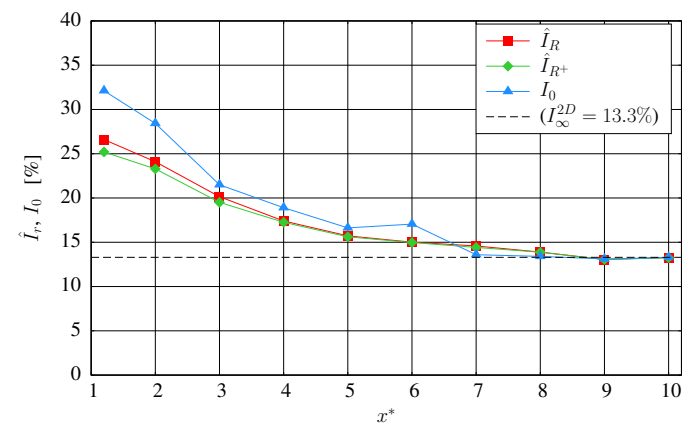

(d) Turbulence intensity $\left(I_{\infty}=15 \%, I_{\infty}^{2 D} \approx 13.3 \%\right)$

Figure 11: Axial velocity deficit (top) and downstream turbulence intensity rate (bottom) in the turbine wake with $U_{\infty}=0.8 \mathrm{~m} \cdot \mathrm{s}^{-1}$ and $I_{\infty}=3 \%$ (left), and with $U_{\infty}=0.83 \mathrm{~m} \cdot \mathrm{s}^{-1}$ and $I_{\infty}=15 \%$ (right).

the maximum available power is proportional to the velocity cubed, all other parameters (fluid density, rotor radius) being constant.

A $20 \%$ mean velocity deficit is however obtained at $x^{*} \approx 3$ for $I_{\infty}=15 \%$, then decreasing to approximately $10 \%$ at $x^{*} \approx 5$ and finally to less than $5 \% 10$ diameters downstream. As a matter of comparison, the power deficit for $I_{\infty}=15 \%$ is approximately $15 \%$ at 10 diameters compared to the $50 \%$ for $I_{\infty}=3 \%$ at the same location. These observations are very important, especially regarding the fact that the turbine performances, in terms of power coefficient $C_{P}$, are only little influenced by the ambient turbulence (Figures $6 \mathrm{a}$ and $6 \mathrm{~b}$ ). This point is a crucial issue in terms of implanting a turbine in an array, which will be treated in Part II [1].

Regarding the turbulence in the turbine wakes for the two studied ambient turbulence intensity rates, Figures 11c and 11d also illustrate most of the characterising features of the wake flow. First, for $I_{\infty}=3 \%$, in the whole wake $\left(0 \leqslant x^{*} \leqslant 10\right.$ for this study), the three indicators $I_{0}, \hat{I}_{R}$ or $\hat{I}_{R^{+}}$are all contained between $10 \%$ and $22 \%$. The minimum value is however 3 to 4 times higher than the ambient level of $I_{\infty}^{2 D}=3.1 \%$, corresponding to $I_{\infty}=3 \%$. From Figure 11c one can also clearly identify the three main characteristics of the flow field. For $x^{*} \leqslant 3, I_{0}$ is very high with $22 \%$ corresponding to the hub wake (cf. Fig. A.13a in Appendix A), precisely located at the wake centre and decaying through diffusion. On the contrary, $\hat{I}_{R}$ or $\hat{I}_{R^{+}}$are rather low owing to the 
fact that, except for the centreline and the thin vortical shear layer issuing from the blade tips, the turbulence intensity is also very low. The merging zone identified earlier (see section 4.1) can also be distinguished between $4 \leqslant x^{*} \leqslant 7$, characterised by a peak in $I_{0} \approx 20 \%$ and the highest values of $\hat{I}_{R}$ and $\hat{I}_{R^{+}}$as well. Those last two indicators increase from $x^{*} \approx 0$ owing to the expansion of the shear layers, until their merging at this location, resulting in these higher values. Finally, from $x^{*} \geqslant 7$, the three indicators become rather similar with $I_{0}$ slightly higher due to a bell shape wake.

Concerning the three indicators for $I_{\infty}=15 \%$, the situation is completely different. As already observed on Figure 9f, the merging of the shear layer appears early in the wake flow, just after the blades and until $x^{*} \approx 3$. This merging zone is characterised, as for $I_{\infty}=3 \%$, by a higher value of $I_{0}\left(I_{0} \approx 32 \%\right.$ at $\left.x^{*}=1.2\right)$ and similar lower values of $\hat{I}_{R}$ and $\hat{I}_{R^{+}}$. From $x^{*} \geqslant 3$, the bell shape profiles are clearly identifiable (cf. Appendix A) and this phenomenon is characterised by the fact that, from $x^{*} \approx 5$, the three indicators tend to collapse into a single curve which is obvious for $x^{*} \geqslant 7$. In addition, from $x^{*} \geqslant 7, I_{0}, \hat{I}_{R}$ or $\hat{I}_{R^{+}}$are identical and equal to the ambient initial value of $I_{\infty}^{2 D}=13.3 \%$ corresponding to $I_{\infty}=15 \%$.

As a matter of conclusion for $I_{\infty}=15 \%$, from Figure $11 \mathrm{~b}$ and $11 \mathrm{~d}$, one can see that for the studied turbine in the studied conditions, after $x^{*} \approx 6$ or 7 , the flow conditions are almost identical to the ambient upstream condition with only $5 \%$ velocity deficit and the recovered ambient turbulence intensity. At this location of the flow, no more coherent flow patterns can be found and, in the case of a potential second turbine downstream, this turbine would apparently experience ambient inflow conditions with a power deficit of only $15 \%$ corresponding to a $5 \%$ velocity deficit. The above described situation will be tested and assessed in Part II [1] of the present study.

\section{Conclusions and prospects}

This study has pointed out the significant role of the ambient turbulence intensity rate on the behaviour of a 3-bladed horizontal axis marine current turbine. First of all, for this precise turbine, the mean performances are hardly influenced by this parameter, which makes it possible to implant turbines with the same design in sites with different turbulence intensity conditions, without too much loss of overall efficiency. In addition, the mean turbine performances should remain stable in time, even if variations of the ambient turbulence conditions occur. However, the performance fluctuations dramatically increase with the turbulence intensity. This may have a major impact on the fatigue of the machine, and thus on its global manufacturing cost [20,27].

The ambient turbulence intensity also has a considerable influence on the turbine wake. As a matter of fact, the wake shape, length and strength largely depend on the upstream turbulence conditions. On the one hand, with $I_{\infty}=3 \%$, the wake remains pronounced even ten diameters downstream of the turbine, with almost $20 \%$ velocity deficit and more than three times the upstream ambient turbulence intensity. Furthermore, the shear layers are still visible, which expresses the presence of coherent vortical structures, and thus the flow homogeneity is still not recovered. On the other hand, with a higher turbulence intensity, $I_{\infty}=15 \%$, the wake dissipates much faster. Indeed, the upstream conditions, in terms of velocity, turbulence intensity and shear stress, are almost fully recovered six diameters downstream, where the flow becomes homogeneous again.

Further studies should now be carried out on the wake characterisation behind a turbine with a lower TSR (e.g., TSR $\approx 3$, for which the loss of power is only about $15 \%$ ) in a $I_{\infty}=3 \%$ turbulence condition. The wake may then be less pronounced, which could allow a closer second turbine implantation. 
As a conclusion, in the context of farm implantation, a second turbine, located at a given distance in the direct wake of the upstream turbine, should recover more energy if the upstream flow has a high turbulence intensity. This high turbulence advantage is counterbalanced by the fact that such conditions also imply more force fluctuation and thus accelerate the machine wear. The issue of interactions between two aligned marine current turbines was also investigated and will be discussed in the second part of this paper [1].

\section{Appendix A. Wake profiles}

Figure A.12 presents axial velocity profiles at several distances downstream of the turbine, for both $I_{\infty}=3 \%$ (Fig. A.12a) and $I_{\infty}=15 \%$ (Fig. A.12b).

Figure A.13 presents downstream turbulence intensity profiles at several distances downstream of the turbine, for both $I_{\infty}=3 \%$ (Fig. A.13a) and $I_{\infty}=15 \%$ (Fig. A.13b). 

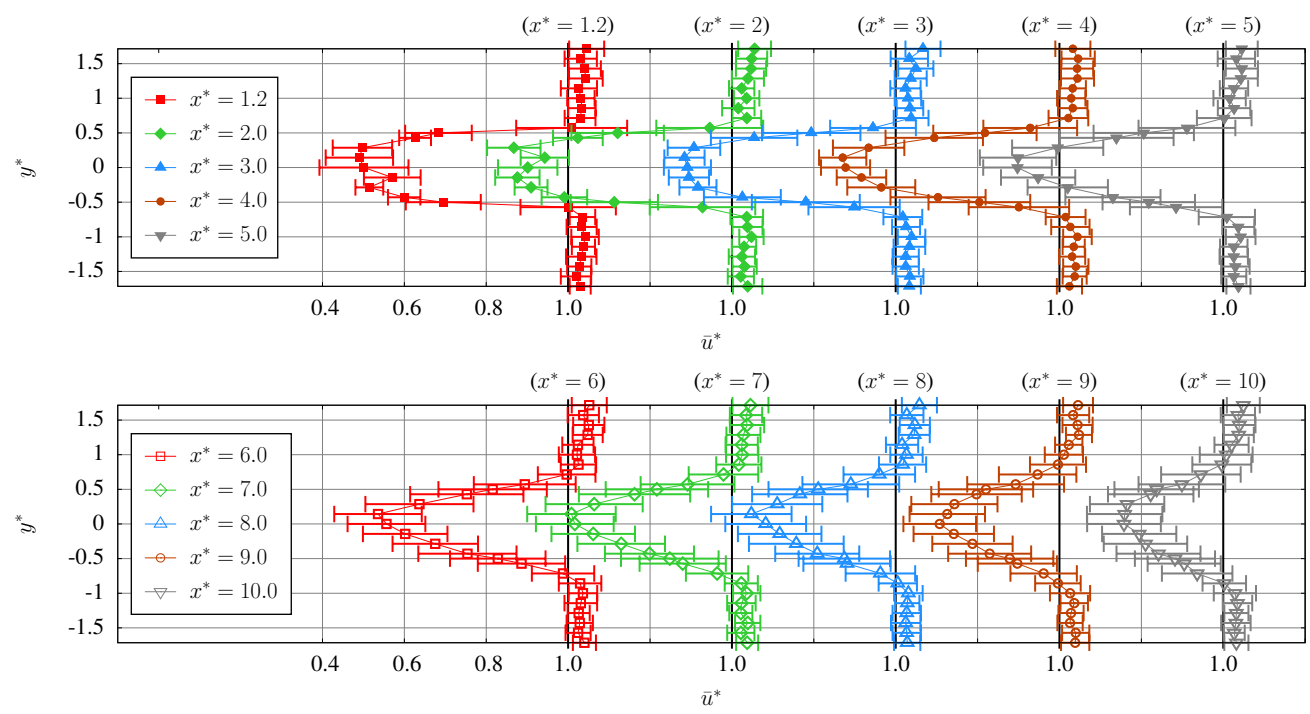

(a) $I_{\infty}=3 \%$.

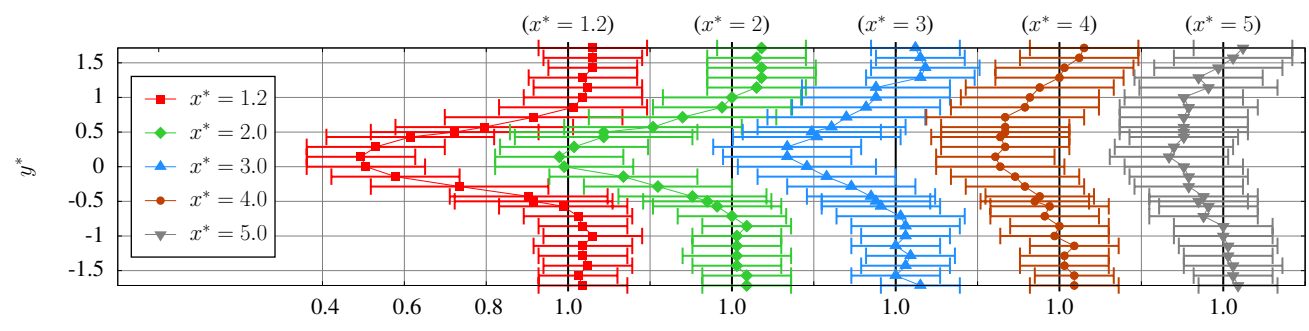

$\bar{u}^{*}$

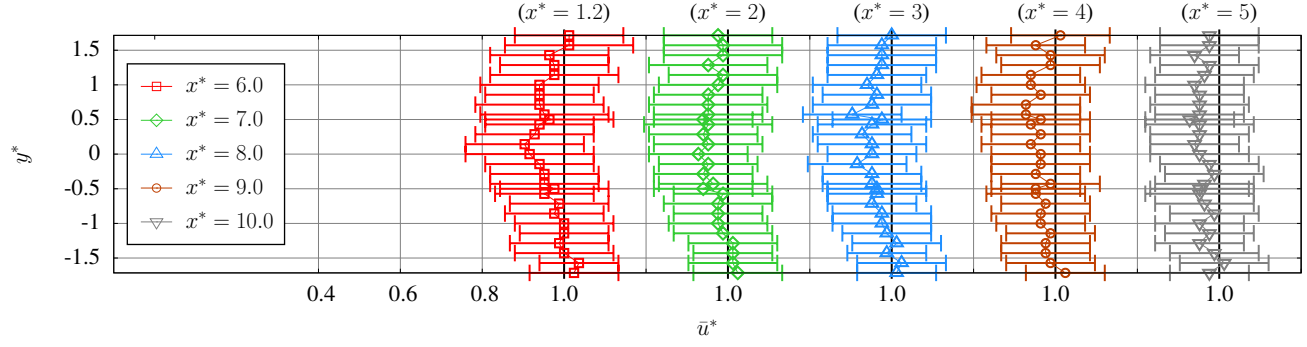

(b) $I_{\infty}=15 \%$.

Figure A.12: Axial velocity profiles with $\mathrm{TSR}=3.67, U_{\infty}=0.8 \mathrm{~m} \cdot \mathrm{s}^{-1}$ and for $I_{\infty}=3 \%$ (top) and $I_{\infty}=15 \%$ (bottom). 

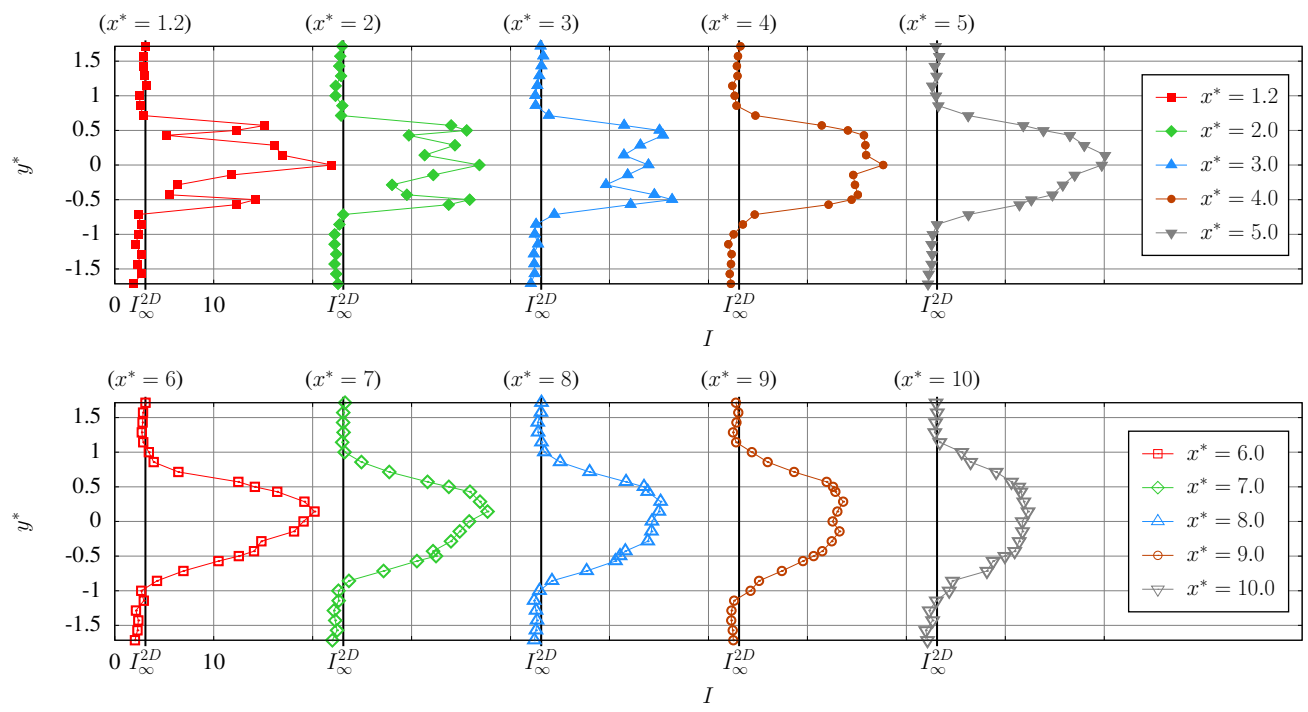

(a) $I_{\infty}=3 \%$.
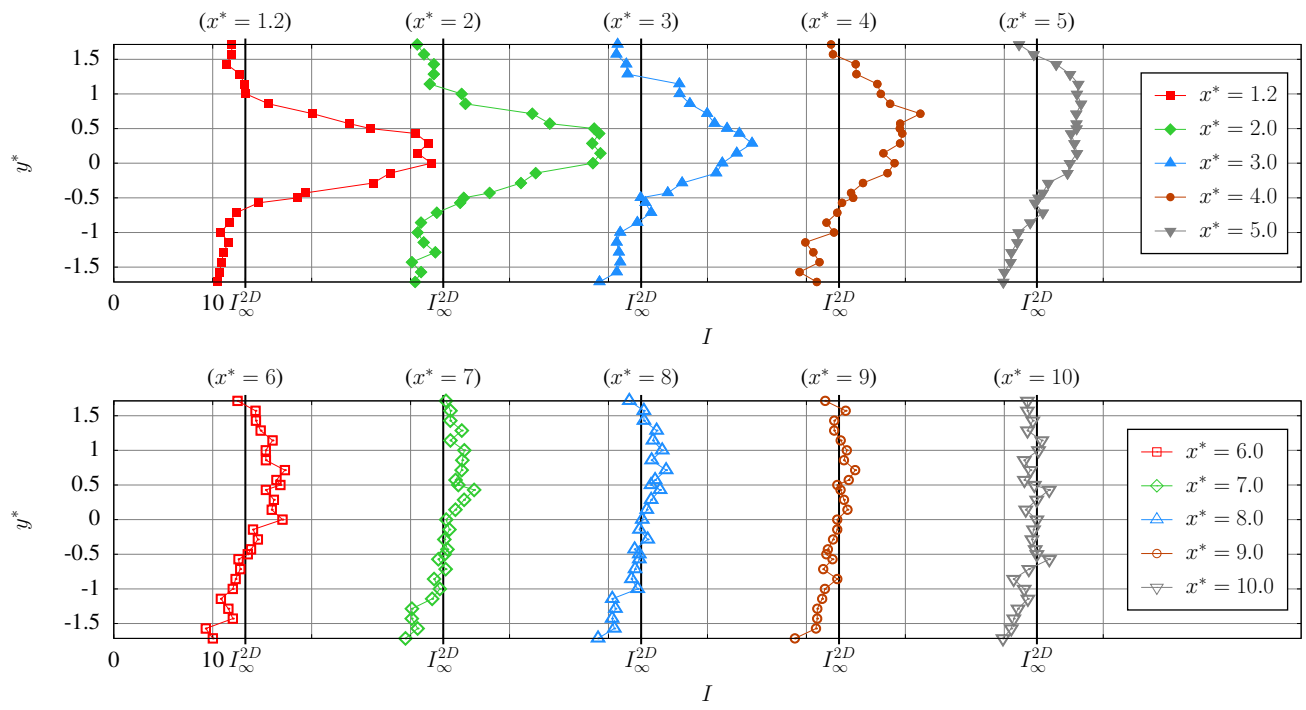

(b) $I_{\infty}=15 \%$.

Figure A.13: Downstream turbulence intensity profiles with $I_{\infty}=3 \%$ (top) and $I_{\infty}=15 \%$ (bottom). 


\section{Appendix B. Convergence results for LDV measurements}

In order to justify the use of $100 \mathrm{~s}$ as a LDV measurement duration, convergence graphs are presented at nine different strategic points downstream of the turbine. Figure B.14 describes their location in the wake.

The TSR and the flow velocity $U_{\infty}$ are those of the previously presented experiments, that is to say TSR $=3.67$ and $U_{\infty}=0.8 \mathrm{~m} \cdot \mathrm{s}^{-1}$ (for $I_{\infty}=3 \%$ ) or $U_{\infty}=0.83 \mathrm{~m} \cdot \mathrm{s}^{-1}$ (for $I_{\infty}=15 \%$ ). Several measured quantities are shown, namely the axial velocity $\bar{u}$, the radial velocity $\bar{v}$, the Reynolds first and second normal stresses $\sigma_{u}^{2}$ and $\sigma_{v}^{2}$, the Reynolds shear stress $\overline{u^{\prime} v^{\prime}}$ and the turbulence intensity rate $I$.

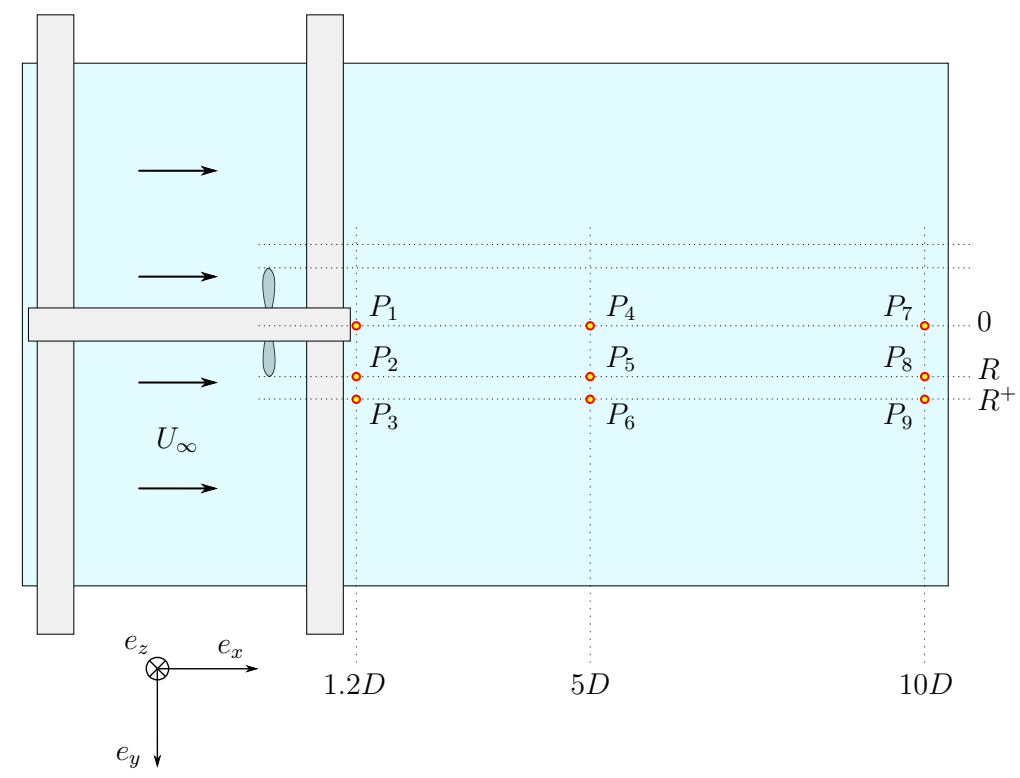

Figure B.14: LDV measurement points for convergence checking.

Figure B.15 presents the convergence results with $I_{\infty}=3 \%$. Time-averaged quantities $\bar{q}$ are plotted against the averaging duration $t$. The graphs show that the average seams to be converged very early, around $t=50 \mathrm{~s}$. Therefore, $T=100 \mathrm{~s}$ is a correct measurement (and thus averaging) duration.

Likewise, Figure B.16 presents the convergence results with $I_{\infty}=15 \%$. The convergence seams to appear a little later, around $t=80 \mathrm{~s} . \quad T=100 \mathrm{~s}$ is then still an appropriate measurement duration. 


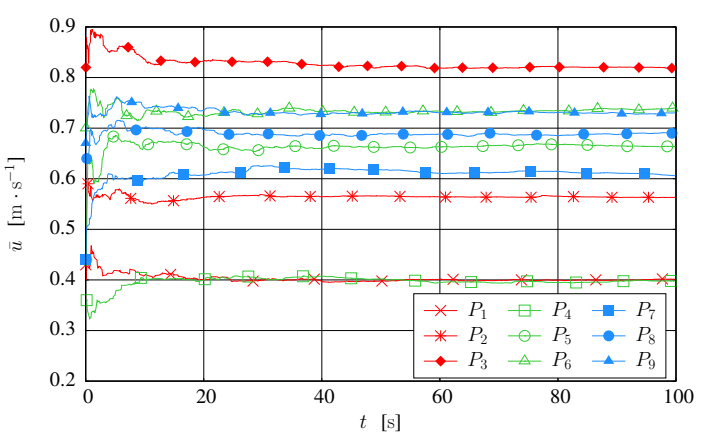

(a) Axial velocity $\bar{u}$

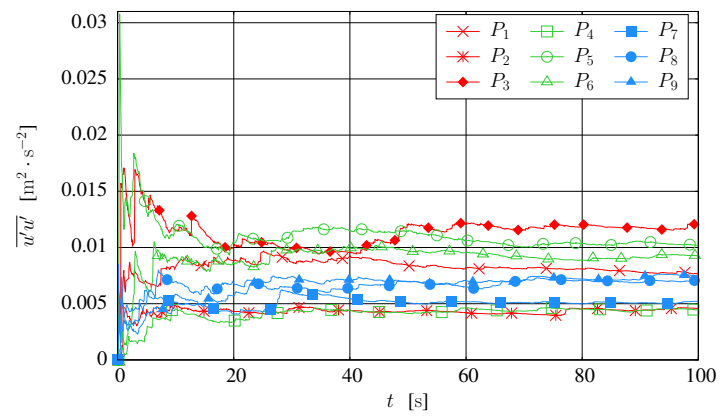

(c) Reynolds first normal stress $\overline{u^{\prime} u^{\prime}}=\sigma_{u}^{2}$

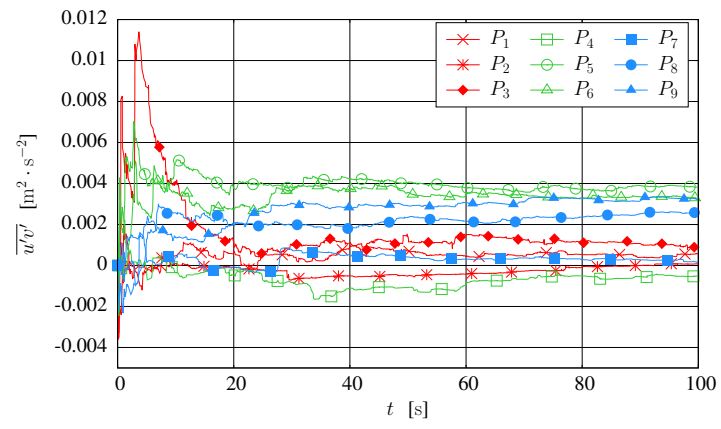

(e) Reynolds shear stress $\overline{u^{\prime} v^{\prime}}$

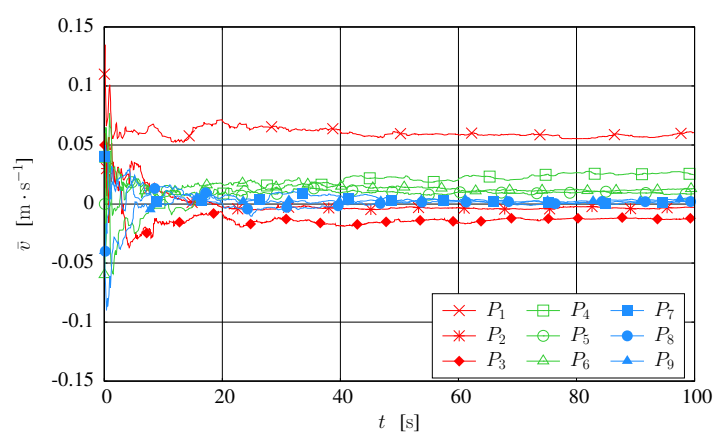

(b) Radial velocity $\bar{v}$

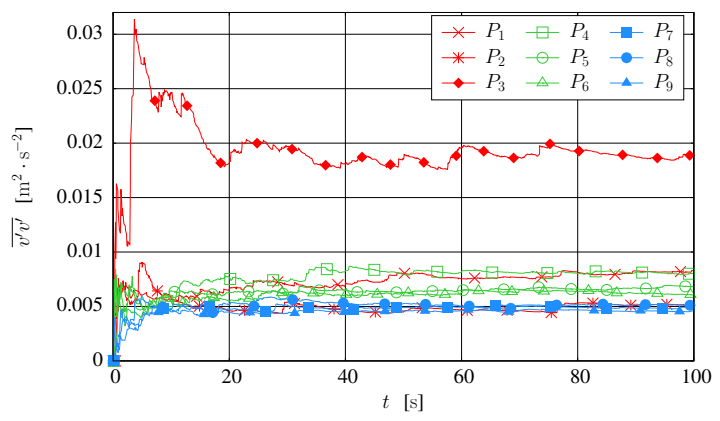

(d) Reynolds second normal stress $\overline{v^{\prime} v^{\prime}}=\sigma_{v}^{2}$

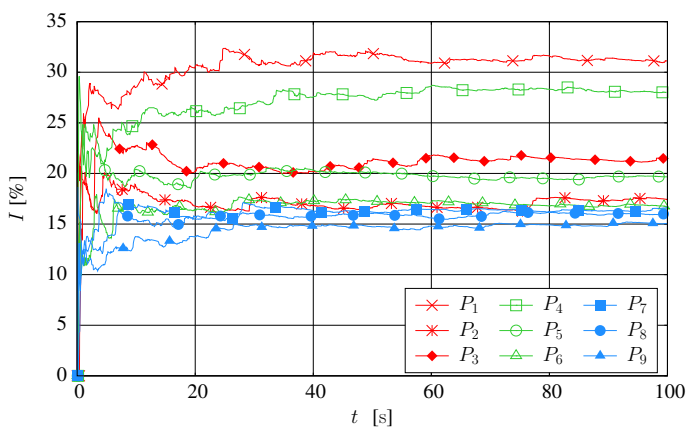

(f) Turbulence intensity rate $I$

Figure B.15: Convergence of various time-averaged quantities as a function of the measurement time $t$, with TSR $=$ $3.67, U_{\infty}=0.8 \mathrm{~m} \cdot \mathrm{s}^{-1}$ and $I_{\infty}=3 \%$. 


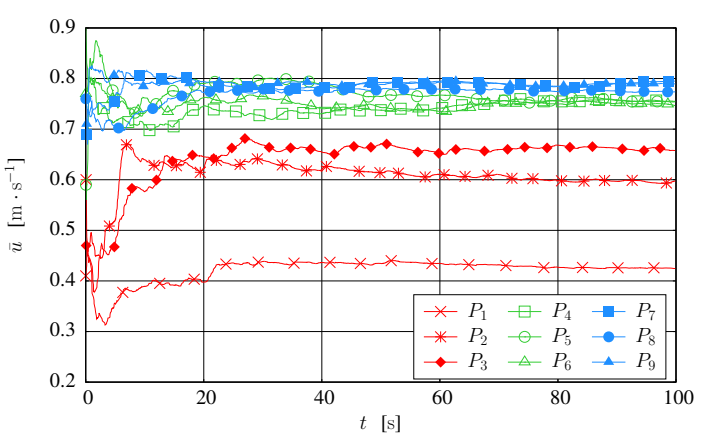

(a) Axial velocity $\bar{u}$

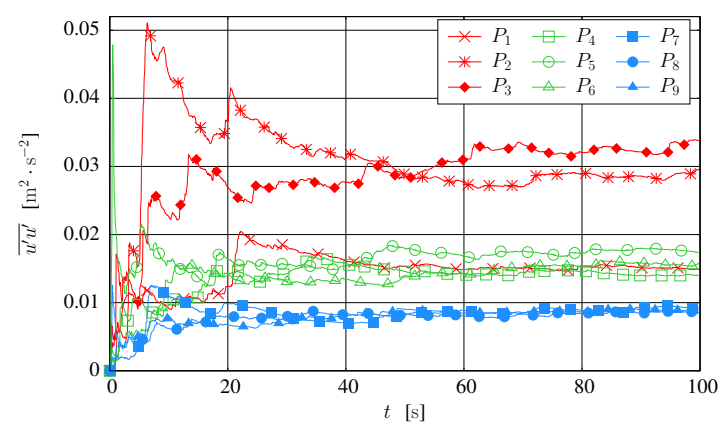

(c) Reynolds first normal stress $\overline{u^{\prime} u^{\prime}}=\sigma_{u}^{2}$

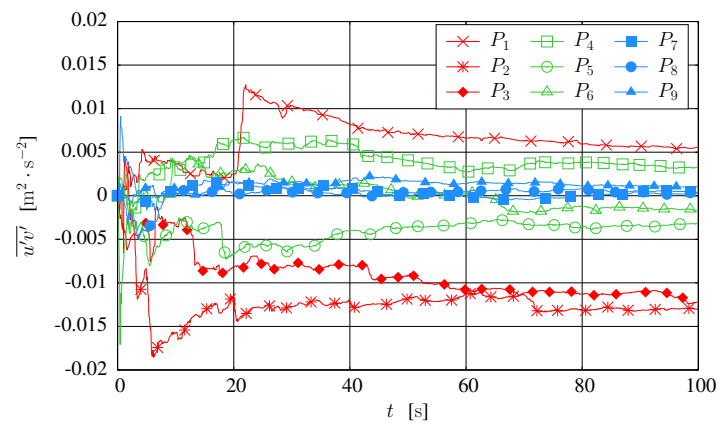

(e) Reynolds shear stress $\overline{u^{\prime} v^{\prime}}$

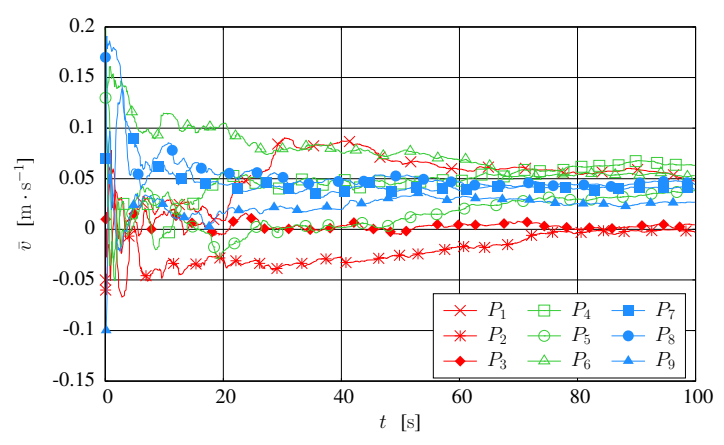

(b) Radial velocity $\bar{v}$

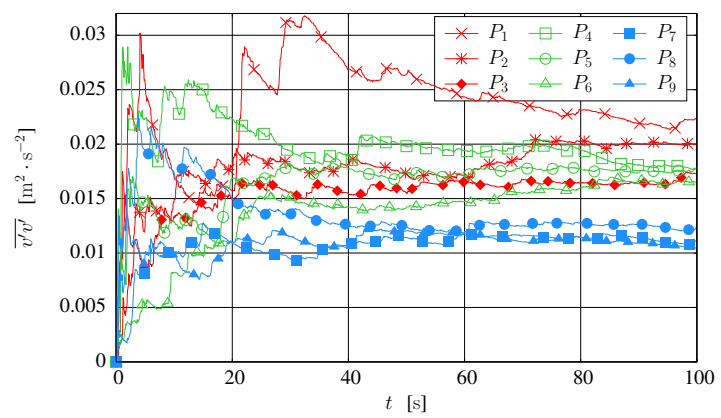

(d) Reynolds second normal stress $\overline{v^{\prime} v^{\prime}}=\sigma_{v}^{2}$

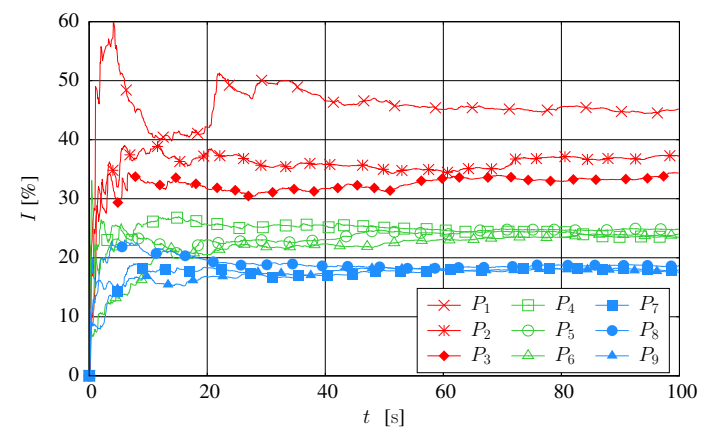

(f) Turbulence intensity rate $I$

Figure B.16: Convergence of various time-averaged quantities as a function of the measurement time $t$, with TSR $=$ $3.67, U_{\infty}=0.83 \mathrm{~m} \cdot \mathrm{s}^{-1}$ and $I_{\infty}=15 \%$. 


\section{Appendix C. Convergence results for force and moment measurements}

A convergence analysis on the force and moment measurements is also presented with $I_{\infty}=15 \%$. Time-averaged $C_{T}$ function of the averaging duration are shown on Figures C.17 and C.18 for different $U_{\infty}$ and TSR conditions. Likewise, Figures C.19 and C.20 present time-averaged $C_{p}$. For both quantities, the convergence seams to be reached from $t=50 \mathrm{~s}$. Again, $T=100 \mathrm{~s}$ is thus an appropriate measurement (and thus averaging) duration.

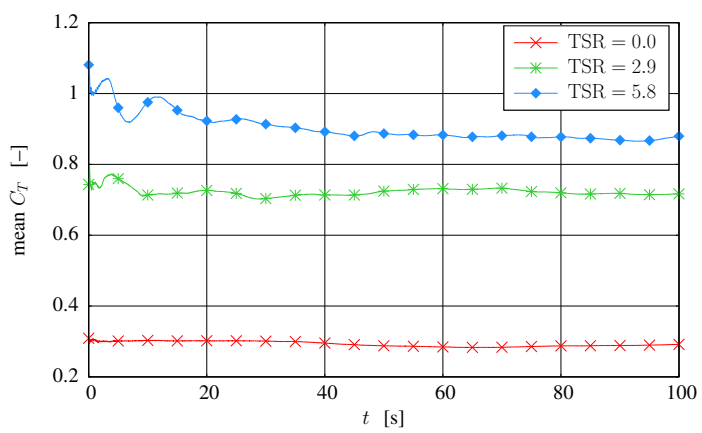

(a) $U_{\infty}=0.41 \mathrm{~m} \cdot \mathrm{s}^{-1}$

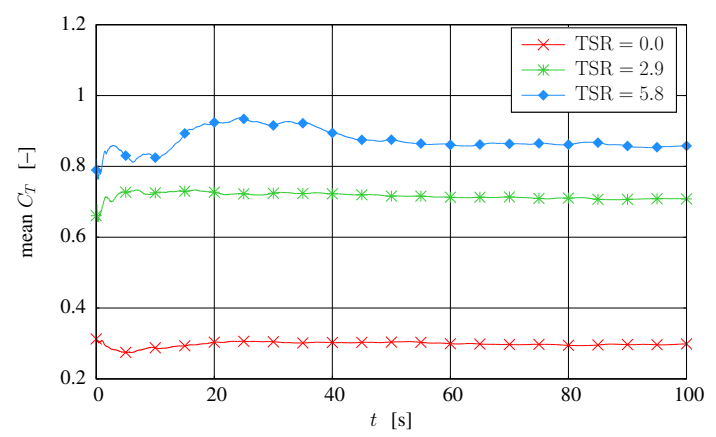

(b) $U_{\infty}=0.83 \mathrm{~m} \cdot \mathrm{s}^{-1}$

Figure C.17: Time-averaged $C_{T}$ convergence as a function of the measurement time $t$ for two given velocities $U_{\infty}=0.41 \mathrm{~m} \cdot \mathrm{s}^{-1}$ (left) and $U_{\infty}=0.83 \mathrm{~m} \cdot \mathrm{s}^{-1}$ (right), with $I_{\infty}=15 \%$. Three different TSR values are considered.

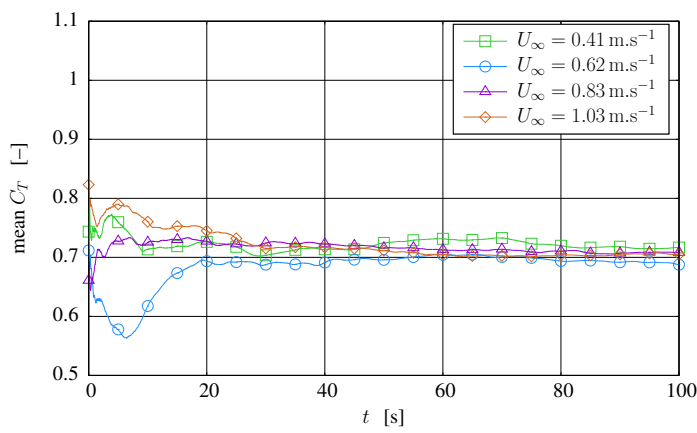

(a) $\operatorname{TSR}=2.9$

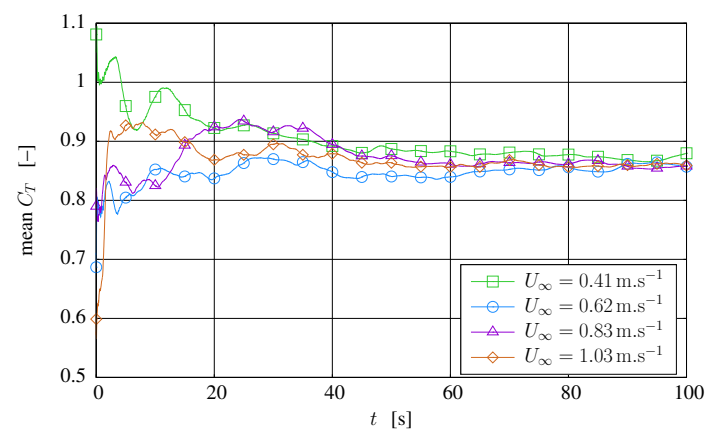

(b) $\mathrm{TSR}=5.8$

Figure C.18: Time-averaged $C_{T}$ convergence as a function of the measurement time $t$ for two given TSR, TSR $=2.9$ (left) and TSR $=5.8$ (right), with $I_{\infty}=15 \%$ and various upstream velocities. 


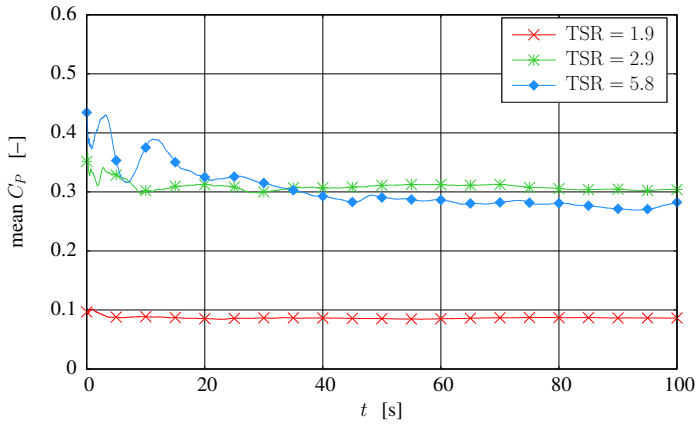

(a) $U_{\infty}=0.41 \mathrm{~m} \cdot \mathrm{s}^{-1}$

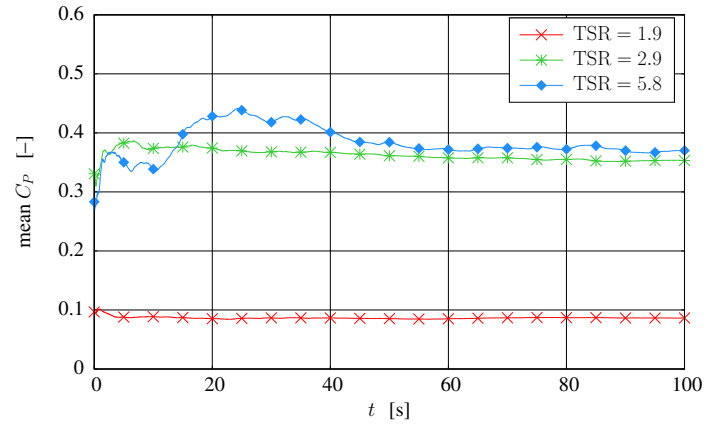

(b) $U_{\infty}=0.83 \mathrm{~m} \cdot \mathrm{s}^{-1}$

Figure C.19: Time-averaged $C_{P}$ convergence as a function of the measurement time $t$ for two given velocities $U_{\infty}=0.41 \mathrm{~m} \cdot \mathrm{s}^{-1}$ (left) and $U_{\infty}=0.83 \mathrm{~m} \cdot \mathrm{s}^{-1}$ (right), with $I_{\infty}=15 \%$. Three different TSR values are considered.

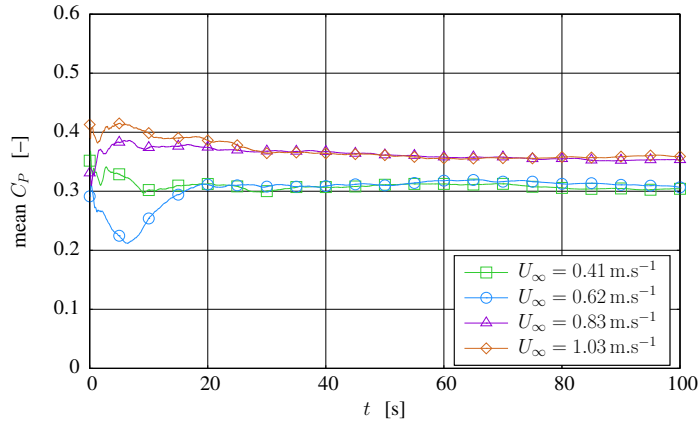

(a) $\operatorname{TSR}=2.9$

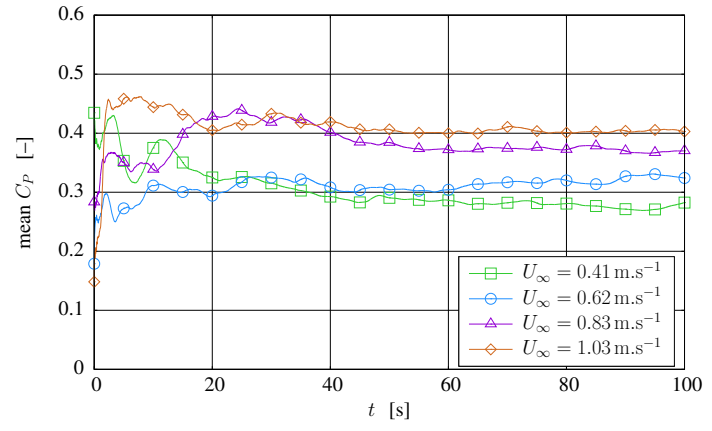

(b) $\operatorname{TSR}=5.8$

Figure C.20: Time-averaged $C_{P}$ convergence as a function of the measurement time $t$ for two given TSR, TSR $=2.9$ (left) and TSR $=5.8$ (right), with $I_{\infty}=15 \%$ and various upstream velocities. 


\section{Appendix D. Spectral analysis}

Figure D.21 shows the frequency spectra of the $C_{T}$ and $C_{P}$ measurements on the turbine TSR = 2 and for two upstream flow conditions. With $I_{\infty}=3 \%$ (Fig. D.21a), the $C_{T}$ spectrum clearly enables to identify the frequency of a blade passing before the mast $f_{b}$, owing to the induced modification of the axial force on the mast. The corresponding $C_{P}$ spectrum enables to identify the rotation frequency $f_{r}$, probably the blades slight imbalance. One can observe that $f_{b} \approx 2.23 \mathrm{~Hz}$ consistently equals three times the rotation frequency $f_{r} \approx 0.74 \mathrm{~Hz}$. The rotation velocity $\left|\Omega_{x}\right|=$ $2 \pi f_{r}$ and thus the TSR can then be deduced thanks to equation (9). Here, the deduced TSR is 2.03 which is close to the nominative (prescribed) value of TSR $=2$. On the other hand, with $I_{\infty}=15 \%$, there is two much noise on both $C_{P}$ and $C_{T}$ signals and no frequency can be easily distinguished. It should be noted that similar and more detailed spectral analyses were carried out by Maganga et al. [21] and Gaurier et al. [27].
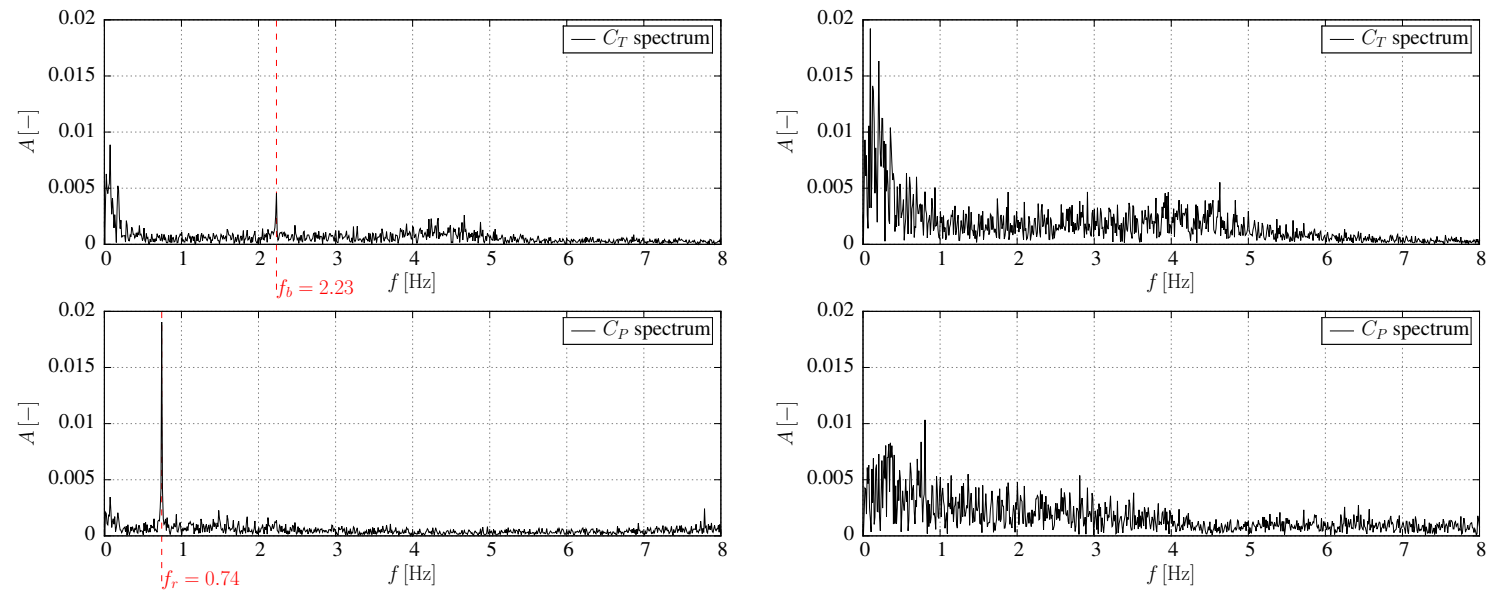

(a) $I_{\infty}=3 \%$

(b) $I_{\infty}=15 \%$

Figure D.21: $C_{T}$ and $C_{P}$ spectra on the turbine with TSR $=2$. Two upstream conditions are considered, namely $I_{\infty}=3 \%$ and $U_{\infty}=0.8 \mathrm{~m} \cdot \mathrm{s}^{-1}$ on the one hand, and $I_{\infty}=15 \%$ and $U_{\infty}=0.83 \mathrm{~m} \cdot \mathrm{s}^{-1}$ on the other hand.

Figure D.22 shows axial velocity fluctuation spectra of the upstream and wake flow. For both turbulence intensity rates, the upstream spectra show that the fluctuations are mostly low frequencies, below $1 \mathrm{~Hz}$. The wake spectra is obtained from measurements at point $P_{2}$ of Figure B.14, that is at right behind the turbine $(x=1.2 D)$, right opposite the blade tip $(y=R)$ where tip vortices emission is likely to occur. The spectra is slightly altered by the presence of the turbines. In addition, the blade passing frequency $f_{b}=4.13 \mathrm{~Hz}$ can be clearly identified with $I_{\infty}=3 \%$, probably thanks to tip vortices. This frequency corresponds to a TSR of 3.78, which is close to the nominative value of $\mathrm{TSR}=3.67$. 

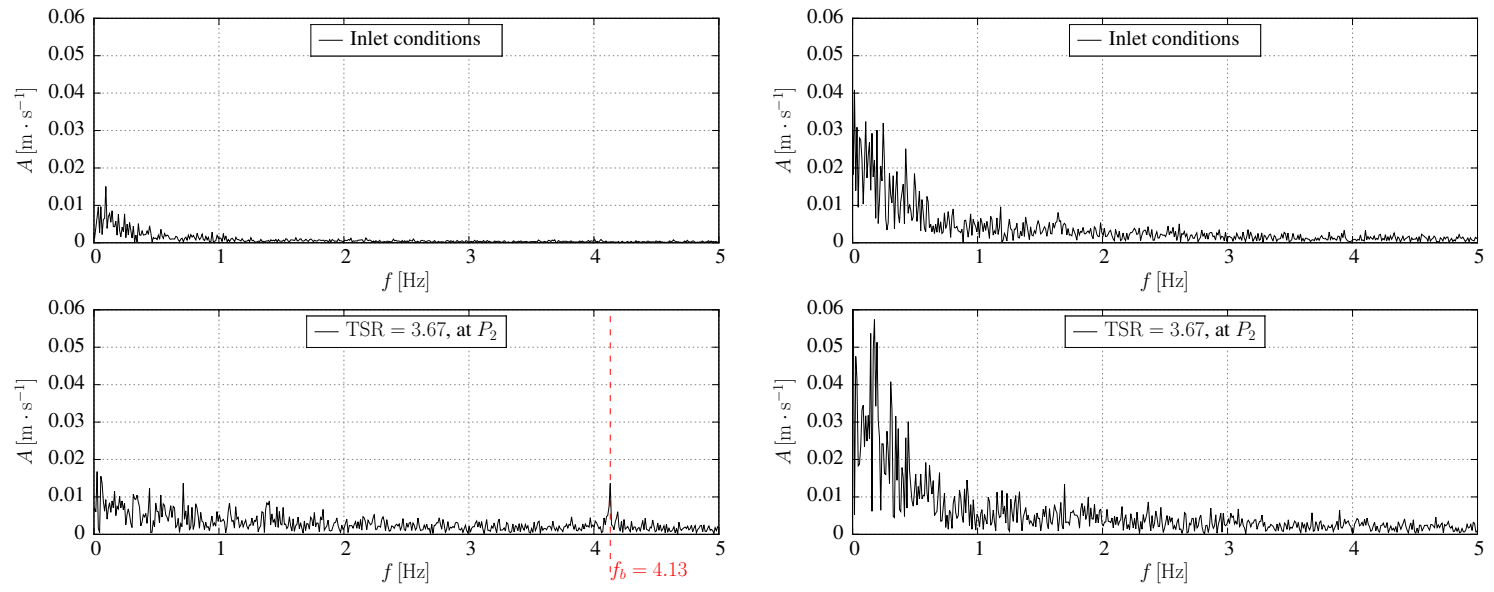

(a) $I_{\infty}=3 \%$

(b) $I_{\infty}=15 \%$

Figure D.22: Axial velocity fluctuation spectra of the upstream $\left(u_{\infty}^{\prime}\right)$ and wake $\left(u^{\prime}\right)$ flow. The wake measurement is performed at point $P_{2}$ of Figure B.14, i.e. at $x=1.2 D$ and $y=R$. Two upstream conditions are considered, namely $I_{\infty}=3 \%$ and $U_{\infty}=0.8 \mathrm{~m} \cdot \mathrm{s}^{-1}$ on the one hand, and $I_{\infty}=15 \%$ and $U_{\infty}=0.83 \mathrm{~m} \cdot \mathrm{s}^{-1}$ on the other hand. 


\section{Acknowledgments}

The authors would like to thank Haute-Normandie Regional Council and IFREMER for their financial support of co-financed PhD theses, as well as the RHYNO project. We are most grateful to Thomas Bacchetti and Jean-Valery Facq for their help in the present work.

\section{References}

[1] P. Mycek, B. Gaurier, G. Germain, G. Pinon, E. Rivoalen, Experimental study of the turbulence intensity effects on marine current turbines behaviour. Part II: two interacting turbines, Submitted to Renewable Energy (2013).

[2] I. A. Milne, R. N. Sharma, R. G. J. Flay, S. Bickerton, Characteristics of the turbulence in the flow at a tidal stream power site, Philosophical Transactions of the Royal Society A: Mathematical, Physical and Engineering Sciences 371 (2013).

[3] I. Nezu, H. Nagakawa, Turbulence in open-channel flows, Rotterdam, The Netherlands: A.A. Balkema, 1993.

[4] E. Osalusi, J. Side, R. Harris, Structure of turbulent flow in emec's tidal energy test site, International Communications in Heat and Mass Transfer 36 (2009) 422-431.

[5] E. Osalusi, J. Side, R. Harris, Reynolds stress and turbulence estimates in bottom boundary layer of fall of warness, International Communications in Heat and Mass Transfer 36 (2009) $412-421$.

[6] J. Thomson, B. Polagye, V. Durgesh, M. Richmond, Measurements of turbulence at two tidal energy sites in Puget Sound, WA, IEEE Journal of Oceanic Engineering 37 (2012) 363-374.

[7] J. MacEnri, M. Reed, T. Thiringer, Influence of tidal parameters on seagen flicker performance, Philosophical Transactions of the Royal Society A: Mathematical, Physical and Engineering Sciences 371 (2013).

[8] Y. Li, J. A. Colby, N. Kelley, R. Thresher, B. Jonkman, S. Hughes, Inflow measurement in a tidal strait for deploying tidal current turbines: Lessons, opportunities and challenges, in: ASME 2010 29th International Conference on Ocean, Offshore and Arctic Engineering, 2010, pp. 569-576. doi:doi:10.1115/OMAE2010-20911.

[9] H. Burchard, P. Craig, J. Gemmrich, H. Vanharen, P. Mathieu, H. Meier, W. Smith, H. Prandke, T. Rippeth, E. Skyllingstad, Observational and numerical modeling methods for quantifying coastal ocean turbulence and mixing, Progress In Oceanography 76 (2008) 399-442.

[10] A. R. Kirincich, S. J. Lentz, G. P. Gerbi, Calculating Reynolds Stresses from ADCP Measurements in the Presence of Surface Gravity Waves Using the Cospectra-Fit Method, Journal of Atmospheric and Oceanic Technology 27 (2010) 889-907.

[11] S. Gooch, J. Thomson, B. Polagye, D. Meggitt, Site characterization for tidal power, in: Oceans 2009, October 26-29, 2009. 
[12] A. Bahaj, A. Molland, J. Chaplin, W. Batten, Power and thrust measurements of marine current turbines under various hydrodynamic flow conditions in a cavitation tunnel and a towing tank, Renewable Energy 32 (2007) $407-426$.

[13] A. Bahaj, W. Batten, G. McCann, Experimental verifications of numerical predictions for the hydrodynamic performance of horizontal axis marine current turbines, Renewable Energy 32 (2007) 2479-2490.

[14] L. Myers, A. Bahaj, Experimental analysis of the flow field around horizontal axis tidal turbines by use of scale mesh disk rotor simulators, Ocean Engineering 37 (2010) 218-227.

[15] M. Harrison, W. Batten, L. Myers, A. Bahaj, Comparison between CFD simulations and experiments for predicting the far wake of horizontal axis tidal turbines, IET Renewable Power Generation 4 (2010) 613-627.

[16] S. Rose, A. Good, M. Atcheson, G. Hamill, C. Johnstone, P. MacKinnon, D. Robinson, A. Grant, T. Whittaker, Investigating experimental techniques for measurement of downstream near wake of a tidal turbine, in: 9th European Wave and Tidal Energy Conference (EWTEC), 2011. Southampton, UK.

[17] T. Stallard, R. Collings, T. Feng, J. Whelan, Interactions between tidal turbine wakes: experimental study of a group of three-bladed rotors, Philosophical Transactions of the Royal Society A: Mathematical, Physical and Engineering Sciences 371 (2013).

[18] S. Tedds, R. Poole, I. Owen, G. Najafian, S. Bode, A. Mason-Jones, C. Morris, T. O’Doherty, D. O'Doherty, Experimental investigation of horizontal axis tidal stream turbines, in: 9th European Wave and Tidal Energy Conference (EWTEC), 2011. Southampton, UK.

[19] I. Milne, A. Day, R. Sharma, R. Flay, Blade loads on tidal turbines in planar oscillatory flow, Ocean Engineering 60 (2013) 163 - 174.

[20] P. Davies, G. Germain, B. Gaurier, A. Boisseau, D. Perreux, Evaluation of the durability of composite tidal turbine blades, Philosophical Transactions of the Royal Society A: Mathematical, Physical and Engineering Sciences 371 (2013).

[21] F. Maganga, G. Germain, J. King, G. Pinon, E. Rivoalen, Experimental characterisation of flow effects on marine current turbine behaviour and on its wake properties, IET Renewable Power Generation 4 (2010) 498-509.

[22] G. Pinon, P. Mycek, G. Germain, E. Rivoalen, Numerical simulation of the wake of marine current turbines with a particle method, Renewable Energy 46 (2012) 111 - 126.

[23] P. Mycek, B. Gaurier, G. Germain, G. Pinon, E. Rivoalen, Numerical and experimental study of the interaction between two marine current turbines, International Journal of Marine Energy 1 (2013). Accepted manuscript.

[24] M. J. Churchfield, Y. Li, P. J. Moriarty, A large-eddy simulation study of wake propagation and power production in an array of tidal-current turbines, Philosophical Transactions of the Royal Society A: Mathematical, Physical and Engineering Sciences 371 (2013). 
[25] G. Germain, Marine current energy converter tank testing practices, in: 2nd International Conference on Ocean Energy (ICOE), 2008. URL: http://archimer .ifremer.fr/doc/00022/ 13366/, Brest, France.

[26] G. Germain, A. S. Bahaj, C. Huxley-reynard, P. Roberts, Facilities for marine current energy converter characterization, in: 7th European Wave and Tidal energy Conference, 2007. URL: http://eprints.soton.ac.uk/75855/.

[27] B. Gaurier, P. Davies, A. Deuff, G. Germain, Flume tank characterization of marine current turbine blade behaviour under current and wave loading, Renewable Energy 59 (2013) 1-12.

[28] S. Wussow, L. Sitzki, T. Hahm, 3d-simulation of the turbulent wake behind a wind turbine, Journal of Physics: Conference Series 75 (2007) 012033. 